\title{
La dynamique protestataire du Mouvement du 20 février à Casablanca
}

\author{
Mounia Bennani-Chraïbi et Mohamed Jeghllaly
}

Le 19 février 2012, le Mouvement du 20 février (M20) fête son premier anniversaire dans plus de 80 localités marocaines. Un an plus tôt, des actions protestataires se sont produites dans 53 sites suite à un appel lancé sur Facebook ${ }^{1}$. Entre temps, les autorités marocaines ont pris des mesures pour désagréger le mouvement, sans pour autant parvenir à mettre fin à la protestation.

A en croire le discours officiel - national et international - qui vante "l'exception marocaine ", la monarchie aurait adopté une voie réformiste qui fait l'économie aussi bien d'une révolution que d'une insurrection populaire. Amorcé dès les années 1990, le « processus de démocratisation » connaîtrait une accélération avec l'adoption d'une nouvelle constitution le $1^{\text {er }}$ juillet 2011, l'organisation d'élections législatives anticipées le 25 novembre 2011, la nomination le 3 janvier 2012 d'un gouvernement sous la présidence du secrétaire général du Parti de la Justice et du Développement $(\mathrm{PJD})^{2}$, jusque-là principale force d'opposition islamiste au sein du Parlement.

Plusieurs interprétations se livrent concurrence pour éclairer la trajectoire marocaine en 2011. Certaines relèvent les facteurs qui font du Maroc un pays « comme les autres », d'autres insistent sur ce qui le singularise. Mais qu'il s'agisse de se pencher sur les situations révolutionnaires ou sur les « exceptions » de la région, quatre biais sont récurrents. En premier lieu, les observateurs tendent à se focaliser sur les « causes » et sur les « résultats » des crises politiques plutôt que sur les dynamiques protestataires ${ }^{3}$. Ensuite, lorsque le regard porte sur les acteurs, il se fixe plutôt sur des « outsiders », incarnés par des archétypes (jeunes déclassés dépolitisés, cyberactivistes, etc.), ignorant ainsi l'hétérogénéité des collectifs. En outre, ces acteurs sont saisis et figés dans un temps « $t$ »; démarche qui occulte les chemins de traverse qui conduisent à protester, à intensifier son engagement, à reconvertir son militantisme ou à se désengager. Enfin, les frontières entre société civile et politique instituée, mouvements sociaux et politique conventionnelle sont rigidifiées ${ }^{4}$; ce qui se traduit par la négligence des processus d'interpénétration des arènes, de formation, de reconfiguration et de désagrégation des coalitions ${ }^{5}$ pendant les crises politiques.

Pour notre part, nous privilégierons un questionnement central : comment un champ d'alliance et d'opposition se configure-t-il en lien avec des événements extérieurs puis se reconfigure-t-il tout long de la dynamique protestataire ? Au prolongement de quelques approches théoriques ${ }^{6}$, le champ d'alliance et d'opposition renvoie à des réseaux d'interactions

\footnotetext{
1 À l'échelle du Maroc, 37000 manifestants d'après la police, 238000 d'après les organisateurs. Dans l'ensemble, le climat est pacifique, exceptés des troubles en fin de journée dans quelques villes (incendies, destructions de biens matériels, 6 morts, 128 blessés dont 115 policiers, et 120 arrestations selon le ministère de l'Intérieur).

${ }^{2}$ Voir tableau 1 et Khadija Mohsen-Finan, Malika Zeghal, «Opposition islamiste et pouvoir monarchique au Maroc. Le cas du Parti de la Justice et du Développement », Revue française de science politique, 56 (1), février 2006, p. 79-119.

${ }^{3}$ Pour une critique, du point de vue des historiens, voir François Furet, Penser la Révolution française, Paris, Gallimard, 1978 ; du point de vue des politistes, voir Michel Dobry, Sociologie des crises politiques, Paris, Presses de Sciences Po, 3éd., 2009.

${ }^{4}$ Pour une critique, voir Jack A. Goldstone, « Introduction. Bridging Institutionalized and Noninstitutionalized Politics », dans Jack A. Goldstone (ed.), States, Parties, and Social Movements, Cambridge, Cambridge University Press, 2003, p. 1-24.

${ }^{5}$ Par exemple, Suzanne Staggenborg, « Coalition Work in the Pro-Choice Movement », Social Problems, 33, 1986, p. 374-389.

${ }^{6}$ Cette notion s'inspire du concept de champ multi-organisationnel (Russell L. Curtis, Louis A. Zurcher, « Stable Resources of Protest Movements : The Multi-Organisational Field », Social Forces, 52 (1), 1973, p. 53-61). Son
} 
plus ou moins stables dans le temps, caractérisés par des relations d'échanges ou d'interconnaissance plus ou moins formalisées ${ }^{7}$, fondées sur le partage ponctuel ou durable de valeurs, de causes, d'adversaires, de participants, de publics, de ressources, etc. Cette notion présente l'intérêt d'articuler : le niveau meso des groupements plus ou moins organisés ; le niveau micro des individus qu'il s'agisse de «nouveaux entrants » ou de multipositionnés qui ont sympathisé/adhéré/participé synchroniquement et/ou diachroniquement à des groupes informels, des groupes de débats sur Facebook, des associations, des syndicats, des partis politiques, des coordinations, etc. Elle permet ainsi de dépasser le biais organisationnel ${ }^{8}$, de restituer la diversité des formes d'organisation, l'hétérogénéité des acteurs qui sont des " participants" plutôt que des "membres", de se saisir du caractère dynamique des " configurations d'alliances changeantes ${ }^{9}$, et d'aller au-delà des frontières habituellement dressées entre mouvements sociaux, organisations partisanes et syndicales, groupements « infrapolitiques ».

Nous essaierons de répondre à ce questionnement à partir de l'observation ethnographique de la coordination du M20 de Casablanca de février 2011 à février 2012. Suite à des appels lancés sur Facebook, le M20 s'est constitué autour d'une plateforme de revendications, d'un esprit pacifique et d'un agenda d'actions protestataires. Ce mouvement multisitué se présente sous forme d'une coordination nationale décentralisée, caractérisée par des «liens faibles " ${ }^{10}$; il regroupe des coordinations fortement imprégnées par les configurations locales dans lesquelles elles s'inscrivent. Dans le sillage du M20, les actions protestataires se démultiplient sur le web et dans l'espace physique de la rue. Nous avons choisi de nous focaliser sur Casablanca. Capitale économique, ville la plus peuplée du Maroc, elle est dotée d'une histoire protestataire inscrite dans les mémoires ${ }^{11}$ et concentre avec Rabat l'essentiel des élites nationales. Bien plus, c'est le fait d'y avoir emmagasiné une expérience de terrain qui nous a conduit à y réaliser plusieurs épisodes d'immersion, variant entre trois jours et un mois, entre avril 2011 et février $2012^{12}$.

\section{Le protocole d'enquête}

Le protocole d'enquête mis en œuvre a obéi à trois impératifs. En premier lieu, il nous a semblé indispensable de multiplier les situations d'observation, synchroniquement et diachroniquement, afin de se saisir de la dynamique protestataire aussi bien dans sa densité qu'en mouvement. Deuxièmement, nous avons souhaité nous donner les moyens de percevoir les effets de cette dynamique sur les participants et sur le collectif. Troisièmement, nous avons veillé à diversifier les modalités de collecte et d'analyse des données de manière à réduire les biais auxquels expose l'usage d'une seule méthode. Nous avons effectué deux focus groups pour affiner notre connaissance de la genèse du M20 à Casablanca. Lors de chaque séjour, nous avons observé les actions protestataires, les assemblées générales et les activités annexes, partagé des moments de sociabilité avec les membres de la coordination, réalisé des entretiens approfondis avec 103 personnes (des participants au M20, des acteurs politiques nationaux, des observateurs, des agents d'autorité), suivi quelques acteurs avec lesquels nous avons réalisé plusieurs entretiens répétés. Pendant les marches, nous avons collecté les tracts, enregistré les

élaboration repose sur l'articulation d'autres concepts (cf. notes infra) et sur une réflexion menée avec Olivier Fillieule.

${ }^{7}$ Voir la notion de «milieu sociopolitique » de Frédéric Sawicki (« Partis politiques et mouvements sociaux », dans Simon Luck, Stéphanie Dechezelles (dir.), Voix de la rue ou voie de urnes ?, Rennes, Presses Universitaires de Rennes, 2011, p. 31-46).

${ }^{8}$ Pamela E. Oliver, « Bringing the Crowd Back In : The Nonorganizational Elements of Social Movements », Research in Social Movements, Conflicts, and Change, 11, 1989, p. 1-30.

${ }^{9}$ Voir le concept d'《entreprises de mouvements sociaux » d'Olivier Fillieule (« De l'objet de la définition à la définition de l'objet. De quoi traite finalement la sociologie des mouvements sociaux ? », Politique et Sociétés, 28 (1), 2009, p. 15-36, dont p. 25 et suiv.).

${ }^{10}$ Mark S. Granovetter, « The Strength of Weak Ties », American Journal of Sociology, 78, 1973, p. 1360-1380.

${ }^{11}$ Les événements de 1965 et de 1981 sont réprimés dans un bain de sang.

${ }^{12}$ M. Bennani-Chraïbi a réalisé 7 sept épisodes d'immersion (avril, juillet, septembre, novembre, décembre 2011, janvier, février 2012), M. Jeghllaly 5 (mai, août, décembre 2011, janvier, février 2012). 
slogans, mené des entretiens courts, pris des photos. En archivant méthodiquement une partie des échanges de membres du M20 sur Facebook, nous avons étendu notre terrain à l'espace d'internet. Enfin, nous avons constitué un dossier de presse, enrichi par les communiqués et les prises de positions des grandes associations, des syndicats, des partis politiques marocains, d'organisations et d'acteurs internationaux.

Afin de construire les séquences de la dynamique protestataire, nous avons procédé à l'analyse méticuleuse de nos données et réalisé un tableau chronologique d'une quarantaine de pages. Celui-ci retrace pendant douze mois les actions menées par le M20 à Casablanca, la variation de la configuration du collectif, les soutiens et les défections à l'échelle nationale, les réponses des autorités, les événements régionaux perçus par nos enquêtés comme favorables ou défavorables au M20, les soutiens internationaux apportés au M20 ou à la monarchie.

Outre l'opportunité de collecter des matériaux d'une grande richesse, enquêter en binôme nous a permis de croiser nos regards, de tisser des relations d'enquête différenciées. En effet, se présenter en tant que jeune chercheur, berbérophone, socialisé dans une université casablancaise, ou en tant qu'enseignante quadragénaire, insérée dans le milieu académique européen n'a pas manqué d'imprégner nos « jeu[x] de distance et de proximité $\gg{ }^{13}$ respectifs.

Sur la base de nos observations, nous émettons deux hypothèses. En premier lieu, c'est la perception d'une " conjoncture fluide $\rangle^{14}$ qui conduit des acteurs à renoncer provisoirement à l'expression de leurs clivages au profit de revendications communes désidéologisées et faiblement hiérarchisées, à se concentrer sur la mise en œuvre d'une «coordination sur le terrain » (tansiq maydani). Deuxièmement, en lien avec les perceptions croisées de ce qui se joue à l'échelle régionale et internationale, les tensions entre espace protestataire et scène politique instituée ${ }^{15}$ contribuent à reconfigurer aussi bien la coalition du M20 que la dynamique protestataire. En partant de ces hypothèses, nous décrirons d'abord le cadre dans lequel se forme une coalition improbable. Nous montrerons que le déclenchement d'un large mouvement de protestation ne résulte ni d'un effet domino, ni de l'avènement d'une génération spontanée ; bien au contraire, l'observation de la genèse du M20 laisse entrevoir la diversité des lieux de gestation, l'intrication des médiations " non relationnelles ${ }^{16}$, informelles et organisées, les synergies entre nouveaux acteurs et militants aguerris. Enfin, nous nous pencherons sur deux processus : celui qui sous-tend l'enracinement de la coalition du M20 et l'extension de la protestation au sein d'un champ d'alliance et d'opposition ; celui qui amorce la reconfiguration, puis la désagrégation de la coalition. Nous défendrons la thèse suivante : les deux processus ne se succèdent pas mécaniquement mais s'interpénètrent dans un « jeu d'échelles » entre le local, le national, le régional et l'international, un faisceau d'actions, d'interactions et d'événements.

\section{Le M20 : à l'intersection entre espace protestataire et scène politique instituée}

Si l'on se réfère à certaines thèses sur l'autoritarisme et sur la formation des coalitions dans les pays du Tiers Monde, la nature du régime marocain présenterait des caractéristiques structurelles peu propices aux soubresauts révolutionnaires. Par opposition aux autoritarismes « exclusifs », « interventionnistes », " répressifs » ${ }^{17}$, le Maroc serait un régime de la "zone grise $»^{18}$. Doté de certains attributs de la démocratie, il demeure imprégné par les « syndromes

\footnotetext{
13 Stéphane Beaud, 1996, «L'usage de l'entretien en sciences sociales. Plaidoyer pour l' "entretien ethnographique », Politix, 9 (35), p. 226-257, dont p. 243.

${ }^{14}$ M. Dobry, Sociologie des crises politiques, op. cit.

${ }^{15}$ Par scène politique instituée nous désignons les lieux de la politique conventionnelle à l'échelle nationale et locale, ainsi que l'ensemble des acteurs admis au sein de ces espaces et dont la participation est encadrée par la loi.

${ }^{16}$ Sur les « voies non relationnelles de diffusion », non fondées sur des liens directs, qui favorisent le processus d' " attribution de similarité », voir l'approche psychosociologique de David Strang, John W. Meyer, « Institutional Conditions for Diffusion », Theory and Society, 22 (4), 1993, p. 487-511.

${ }^{17}$ Misagh Parsa, States, Ideologies \& Social Revolutions. A Comparative Analysis of Iran, Nicaragua and the Philippines, Cambridge, Cambridge University Press, 2000.

18 Thomas Carothers, « The End of the Transition Paradigm », Journal of Democracy, 1, 2002, p. 5-21.
} 
» de « pluralisme irresponsable » ou de « pouvoir politique dominant », qui se traduisent entre autres par une cassure entre gouvernants et gouvernés, par une désaffection générale de ces derniers à l'égard de la politique instituée et des élites. Sur un autre plan, les caractéristiques de son espace protestataire constitueraient un obstacle au déferlement d'une vague révolutionnaire. Le fait qu'une partie de l'opposition puisse espérer un accès même partiel aux institutions étatiques briserait un tel élan. En outre, selon M. Parsa, la présence de challengers fortement idéologisés et trop organisés suscitent de telles craintes, notamment au sein des classes supérieures, qu'elle empêche la formation des coalitions transclassistes. Or dans le cas marocain, d'une part, la monarchie a tenté de canaliser les opposants vers la politique instituée. D'autre part, il existe une perception vivace parmi les protestataires d'une polarisation idéologique et d'un déséquilibre organisationnel entre des islamistes puissants et une gauche radicale faiblement enracinée. Reste à savoir comment des acteurs sont parvenus à former une coalition relativement large pour s'opposer au «despotisme » et à la «corruption » dans un contexte présentant autant d'entraves?

\section{La scène politique instituée marocaine : entre fragmentation et renouvellement}

La monarchie marocaine a souvent été présentée comme une «experte en survie ${ }^{19}$, échappant aux vicissitudes du temps régional en s'ajustant aux transformations de son environnement, en observant les erreurs de ses voisins pour mieux anticiper. Grâce à une stratégie « makhzénienne $»^{20}$, elle assurerait le renouvellement continuel aussi bien du discours de la réforme ${ }^{21}$ que de réseaux de clientèles, elle ferait preuve d'une aptitude à « diviser pour mieux régner », à transformer les « opposants à Sa Majesté » en « opposants de Sa Majesté », voire en «amis du roi»; la scène politique instituée étant caractérisée par un «trop plein d'acteurs $»^{22}$, le recours à la répression demeurerait sélectif et ponctuel.

Dès la proclamation de l'indépendance en 1956, l'un des enjeux de la royauté aurait été de fragmenter un champ politique polarisé en favorisant les scissions et en accompagnant la naissance de partis dits «administratifs ${ }^{23}$. Ainsi, le pluripartisme et les mécanismes électoraux auraient été pensés comme un « instrument de contrôle de la classe politique et de cantonnement des concurrents potentiels ${ }^{24}$. En effet, après avoir été alliés dans la lutte nationaliste, monarchie et Mouvement national ne tardent pas à entrer en concurrence. À partir du milieu des années 1960, les aspirations révolutionnaires s'amplifient au sein de la gauche, l'antimonarchisme se manifeste sous forme de révolte urbaine (1965), de guérilla, de tentatives de coups d'État militaires $(1971,1972)$. C'est le début des « années de plomb », une phase de répression massive. Pour autant, les négociations entre la monarchie et l'aile gauche du Mouvement national se poursuivent et la mobilisation du registre nationaliste à travers l'affaire du Sahara (1975) amorce le début de stabilisation du régime et du processus dit démocratique. La reconnaissance de l'hégémonie du roi, de la marocanité du Sahara et de l'islam comme

\footnotetext{
${ }^{19}$ Lisa Anderson, « Dynasts and Nationalists: Why Monarchies Survive ?” dans J. Kostiner (ed.), Middle East Monarchies. The Challenge of Modernity, Boulder, Lynne Rienner Publishers, 2000.

${ }^{20} \mathrm{Au}$ Maroc, le terme Makhzen désigne la Maison royale, le territoire sur lequel s'étend son pouvoir ainsi que ses extensions administratives. « Makhzenisation » évoque la cooptation par le « Makhzen », ou l'imprégnation par son esprit et son style.

${ }^{21}$ Voir entre autres Myriam Catusse, « Maroc : un fragile État social dans la réforme néolibérale », dans Myriam Catusse, Blandine Destremau, Éric Verdier (dir.), L'État face aux « débordements » du social au Maghreb. Formation, travail, protection, Paris, Karthala, 2010, p. 121-148.

${ }^{22}$ Abdellah Tourabi, Lamia Zaki, « Maroc : une révolution royale ? », Mouvements, 66, 2011, p. 98-103 ; Lofti Chawqui, «Le mouvement du 20 février un an après », Centre Tricontinental (CETRI), le 2 février 2012, $<$ http://www.cetri.be/spip.php?page=imprimer\&id article=2506 $>$.

${ }^{23}$ Rémy Leveau, Le fellah marocain défenseur du trône, Paris, Presses de Sciences Po, 1985 ( $1^{\text {re }}$ éd. : 1976).

${ }^{24}$ Mounia Bennani-Chraïbi, " "Hommes d'affaires" versus "profs de fac". La notabilisation parlementaire d'un parti de militants au Maroc », Revue internationale de politique comparée, 15 (2), 2008, p. 205-219, dont p. 206.
} 
domaine réservé du commandeur des croyants trace les frontières entre l'opposition légale, représentée par l'Union socialiste des forces populaires (USFP) ${ }^{25}$, et les mouvements plongés dans l'illégalité (d'abord d'obédience marxiste, puis islamiste).

Après une décennie marquée par les politiques d'ajustement structurel et les révoltes urbaines (1981, 1984, 1990), avec pour arrière-plan les promesses de démocratisation du côté de la Tunisie (1987) et de l'Algérie (1988), la guerre du Golfe (1991) constitue un moment intense de défi au roi. Cependant, à l'heure des désenchantements, les déboires du voisin algérien auraient incité les différents acteurs politiques à l'autolimitation. Dans un contexte d'appréhension de l'islamisme aussi bien par la monarchie que par les héritiers du Mouvement national, l'ouverture politique se traduit par la relance des négociations entre les deux parties et par la libération progressive de victimes des années de plomb. Parmi ces derniers, nombreux sont ceux qui contribuent à donner un nouvel élan à l'espace associatif et/ou qui renforcent les rangs d'organisations de la gauche radicale peu à peu légalisées ${ }^{26}$.

Quelles que soient les grilles de lecture privilégiées, l' «alternance consensuelle» gouvernementale (1998) et la succession monarchique (1999) sont plus ou moins perçues comme un tournant ${ }^{27}$ : intégration partielle des islamistes au sein du parlement (1997), relative liberté de ton dans la presse, création de l'Instance équité et réconciliation (2004-2006) en vue de tourner la page des années de plomb. Toutefois, suite aux attentats du 16 mai 2003, les autorités renouent avec les pratiques répressives et recadrent le champ médiatique. Depuis 1998 , la prolifération des partis continue à élargir les frontières de la scène politique légale ${ }^{28}$. Peu à peu, l'ingénierie électorale prend le relais du bourrage des urnes : aucun parti politique n'obtient plus de $11 \%$ des voix pendant les législatives de 2007 et le taux d'abstention atteint le record de $63 \%$. Les gouvernements hétéroclites aux prérogatives floues rassemblent des technocrates, des ministres issus des partis du Mouvement national et des anciens partis dits administratifs.

Dès 2008, le Parti authenticité et modernité (PAM) est créé, officieusement, pour contrecarrer la montée électorale des islamistes, officiellement, en vue de réconcilier les citoyens avec la politique en rassemblant les forces vives du royaume. Le fait que celui-ci soit cofondé par un « ami du roi », ancien secrétaire d'État à l'Intérieur, sa progression électorale fulgurante, l'attrait qu'il exerce sur les élus d'anciens partis administratifs conduisent ses adversaires à l'accuser d'être un nouveau « parti administratif », dont la mission serait de parachever le verrouillage de la scène politique instituée.

C'est sur cette toile de fond que la dynamique protestataire impulsée par le M20 vient brouiller les frontières entre politique instituée et espace protestataire.

\section{Le 20 février : une convergence d'acteurs hétéroclites}

Les premières manifestations du M20 qui se produisent le dimanche 20 février 2011, à travers l'ensemble du pays, constituent un seuil inédit dans l'histoire protestataire du Maroc indépendant. Elles rassemblent de nouveaux entrants et des témoins de conflits qui remontent aux « années de plomb », des acteurs associatifs, des adhérents de partis gouvernementaux, de l'opposition parlementaire et des militants d'organisations non légalisées. Certes, l'occupation de la rue s'est quasi routinisée au cours des vingt dernières années et l'espace protestataire s'est

\footnotetext{
${ }^{25}$ Voir tableau 1.

${ }^{26}$ Éric Cheynis, «L'espace des transformations de l'action associative au Maroc. Réforme de l'action publique, investissements militants et légitimation internationale », thèse de doctorat de sciences sociales, Paris, Université Paris I-Panthéon Sorbonne, 2008.

${ }^{27}$ Myriam Catusse, Frédéric Vairel, « Ni tout à fait le même, ni tout à fait un autre. Métamorphoses et continuité du régime marocain », Maghreb-Machrek, 175, 2003, p. 73-92.

${ }^{28}$ Une telle tendance se traduit également dans l'augmentation du nombre des partis représentés au sein de la Chambre des représentants : 3 en $1963 ; 6$ en $1977 ; 8$ en $1984 ; 11$ en $1993 ; 15$ en $1997 ; 21$ en 2002 ; 24 en 2007.
} 
densifié y compris dans les villes moyennes et petites. En 1991, des diplômés chômeurs sans appartenance politique, de gauche, d'extrême gauche et islamistes renoncent à s'affronter pour construire une cause commune autour du droit au travail et enclenchent un cycle de mobilisations qui perdure en se recomposant ${ }^{29}$. Parallèlement, pendant les années 2000, les causes «arabes" ou « islamiques » favorisent des coordinations par-delà les clivages idéologiques. Au cours des dernières années, des coordinations comme celles contre la cherté de la vie réunissent des acteurs associatifs, syndicaux et partisans de la gauche et de l'extrême gauche. Néanmoins, c'est la première fois qu'une tentative de dépasser la fragmentation du champ politique marocain s'inscrit dans un registre de politique nationale ; ce qui n'exclut ni l'imbrication avec des revendications qualifiées de sociales ni les articulations entre les échelles du transnational, du national et du local.

En effet, une jonction a priori improbable s'établit entre des réseaux politiques pour le moins distincts : islamiste et de gauche. Bien davantage, au sein et aux abords mêmes de ces réseaux, protester ensemble nécessite de mettre en sourdine, ne serait-ce que provisoirement, les animosités et la méfiance qui traversent aussi bien la gauche que les islamistes et qui opposent: « organisés » et "indépendants », ceux qui sont intégrés au sein de la politique instituée et ceux qui en sont exclus ou qui y occupent une position marginale (Tableau 1), sans compter les lignes de fracture qui secouent des partis tiraillés entre leur passé et leur présent.

\section{Tableau 1 : Les organisations politiques secouées ou impliquées dans le M20}

\begin{tabular}{|c|c|c|c|c|c|}
\hline \multicolumn{6}{|c|}{ Les organisations politiques secouées par le M20 } \\
\hline \multirow[t]{2}{*}{$\begin{array}{l}\text { Gauche } \\
\text { gouvernementale }\end{array}$} & \multicolumn{2}{|c|}{$\begin{array}{l}\text { Union socialiste des } \\
\text { forces populaires (USFP) }\end{array}$} & \multicolumn{2}{|c|}{$\begin{array}{l}\text { Issu d'une scission du Mouvement } \\
\text { national en 1959, puis d'une autre en } \\
\text { 1975, il incarne l'opposition de gauche } \\
\text { au sein du parlement jusqu'en } 1997 . \\
\text { Dans le cadre de la Koutla (bloc } \\
\text { constitué avec les héritiers du } \\
\text { Mouvement national), il devient un } \\
\text { parti gouvernemental entre } 1998 \text { et } \\
\text { 2011. Son histoire est parsemée de } \\
\text { scissions. }\end{array}$} & $\begin{array}{l}\text { En } 1^{\text {re }} \text { position } \\
\text { pendant les } \\
\text { législatives de } \\
1997 \text { et en } 2002, \\
\text { il passe à la 5e en } \\
\text { nombre de sièges } \\
\text { en } 2007 \text { (38). } \\
\text { Près de } 60000 \\
\text { adhérents } \\
\text { déclarés }\end{array}$ \\
\hline & \multicolumn{2}{|c|}{$\begin{array}{l}\text { Parti du progrès et du } \\
\text { socialisme (PPS) }\end{array}$} & \multicolumn{2}{|c|}{$\begin{array}{l}\text { Il a une filiation avec le Parti } \\
\text { communiste marocain créé en } 1944 . \\
\text { Dans le cadre de la Koutla, il devient } \\
\text { un parti gouvernemental à partir de } \\
1998 .\end{array}$} & $\begin{array}{l}17 \text { sièges au } \\
\text { Parlement en } \\
2007 . \\
\text { Près de } 40000 \\
\text { adhérents } \\
\text { déclarés }\end{array}$ \\
\hline $\begin{array}{l}\text { Opposition } \\
\text { parlementaire }\end{array}$ & \multicolumn{2}{|c|}{$\begin{array}{l}\text { Parti de la justice et du } \\
\text { développement (PJD) }\end{array}$} & \multicolumn{2}{|c|}{$\begin{array}{l}\text { Parti à référent islamiste créé en } 1998 \text {, } \\
\text { après un long processus d'unification } \\
\text { et de légalisation. Il représente la } \\
\text { principale force d'opposition au sein } \\
\text { du parlement jusqu'en } 2011 \text {. }\end{array}$} & $\begin{array}{l}46 \text { sièges au } \\
\text { Parlement en } \\
2007 . \\
\text { Près de } 16000 \\
\text { adhérents } \\
\text { déclarés }\end{array}$ \\
\hline \multicolumn{6}{|c|}{ Les organisations politiques qui soutiennent le M20 } \\
\hline $\begin{array}{l}\text { Opposition } \\
\text { légale } \\
\text { parlementaire et } \\
\text { non } \\
\text { parlementaire : }\end{array}$ & $\begin{array}{l}\text { Opposition } \\
\text { parlementaire } \\
\text { Alliance de la } \\
\text { gauche } \\
\text { démocratique } \\
(2007)\end{array}$ & $\begin{array}{l}\text { Parti so } \\
\text { unifié ( }\end{array}$ & $\begin{array}{l}\text { aliste } \\
\text { UU) }\end{array}$ & $\begin{array}{l}\text { Créé en } 2005 \text {, suite à un } \\
\text { processus d'unification de la } \\
\text { gauche non gouvernementale, il } \\
\text { puise ses racines dans le } \\
\text { Mouvement national et dans les } \\
\text { mouvements marxistes des } \\
\text { années } 1970 \text {. }\end{array}$ & $\begin{array}{l}6 \text { sièges au } \\
\text { Parlement en } \\
2007 . \\
\text { PSU : près de } \\
4000 \text { adhérents } \\
\text { déclarés, } \\
\text { PADS : près de }\end{array}$ \\
\hline
\end{tabular}

\footnotetext{
${ }^{29}$ Montserrat Emperador Badimon, « Les mobilisations des diplômés chômeurs au Maroc : usages et avatars d'une protestation pragmatique », thèse de doctorat en science politique, Aix-en-Provence, Institut d'études politiques, septembre 2011.
} 


\begin{tabular}{|c|c|c|c|c|}
\hline \multirow[t]{3}{*}{$\begin{array}{l}\text { Rassemblement } \\
\text { de la gauche } \\
\text { démocratique } \\
(2004)\end{array}$} & & $\begin{array}{l}\text { Parti de } \\
\text { l'avant-garde } \\
\text { démocratique } \\
\text { socialiste } \\
\text { (PADS) }\end{array}$ & $\begin{array}{l}\text { Issu d'une scission en } 1983 \text { au } \\
\text { sein de l’USFP, légalisé en } \\
1993 .\end{array}$ & \multirow[t]{2}{*}{$\begin{array}{l}1100 \text { adhérents } \\
\text { déclarés, } \\
\text { CNI : information } \\
\text { non disponible. }\end{array}$} \\
\hline & & $\begin{array}{l}\text { Congrès } \\
\text { national } \\
\text { Ittihadi }(\mathrm{CNI})\end{array}$ & $\begin{array}{l}\text { Issu d'une scission en } 2001 \\
\text { d'une partie de l'aile syndicale } \\
\text { de l'USFP (Confédération } \\
\text { démocratique du travail- CDT). }\end{array}$ & \\
\hline & \multicolumn{2}{|c|}{$\begin{array}{l}\text { Opposition légale, non } \\
\text { parlementaire } \\
\text { Annahj Addimocrati }\end{array}$} & $\begin{array}{l}\text { Créé en 1995, légalisé en } 2004 \text {, } \\
\text { ce parti se réclame de Ila Al } \\
\text { Amam, mouvement marxiste- } \\
\text { léniniste des années } 1970 .\end{array}$ & $\begin{array}{l}\text { Tradition de } \\
\text { boycott des } \\
\text { élections. } \\
\text { Près de } 1000 \\
\text { adhérents } \\
\text { déclarés. }\end{array}$ \\
\hline \multirow[t]{6}{*}{$\begin{array}{l}\text { Organisations } \\
\text { politiques non } \\
\text { reconnues par } \\
\text { les autorités }\end{array}$} & \multirow[t]{3}{*}{ Islamistes } & $\begin{array}{l}\text { Al Adl Wal Ihsane } \\
\text { (Justice et } \\
\text { spiritualité) }\end{array}$ & \multicolumn{2}{|c|}{$\begin{array}{l}\text { L'une des principales organisations islamistes } \\
\text { marocaines dont la gestation a commencé au milieu } \\
\text { des années } 1970 \text {. Elle se distingue par sa non- } \\
\text { reconnaissance du statut de commandeur des } \\
\text { croyants au roi et par l'importance qu'elle accorde à } \\
\text { l'éducation spirituelle. }\end{array}$} \\
\hline & & $\begin{array}{l}\text { Al Badil Al Hadari } \\
\text { (Alternative } \\
\text { civilisationnelle) }\end{array}$ & \multirow{2}{*}{\multicolumn{2}{|c|}{$\begin{array}{l}\text { Issus de la même matrice islamiste des années } 1970 \\
\text { que le PJD, ils se proclament « islamo-démocrates » } \\
\text { et proches de la gauche. Le premier est légalisé en } \\
2005 \text {, puis dissout en } 2008 \text { suite à des accusations de } \\
\text { terrorisme. Le second n'a pas été officialisé avant } \\
2012 \text {. }\end{array}$}} \\
\hline & & Hizb Al Oumma & & \\
\hline & \multirow{3}{*}{$\begin{array}{l}\text { Extrême } \\
\text { gauche }\end{array}$} & Maoïstes & \multirow{3}{*}{\multicolumn{2}{|c|}{$\begin{array}{l}\text { Présents essentiellement au sein de quelques } \\
\text { universités }\end{array}$}} \\
\hline & & $\begin{array}{l}\text { Trotskystes } \\
\text { (Almounadil-a) }\end{array}$ & & \\
\hline & & Stalinistes & & \\
\hline
\end{tabular}

Tableau 2 - Principales appartenances associatives des pionniers de "gauche 》 du M20 Casablanca

\begin{tabular}{|l|l|}
\hline Association marocaine des droits humains (AMDH) & $\begin{array}{l}\text { Créée en 1979 dans le giron de l'USFP. Après } \\
\text { une phase de mise en veille, dès 1988, elle connaît } \\
\text { un renouveau avec l'affluence de militants de la } \\
\text { gauche radicale. En 2009, elle compte 91 sections } \\
\text { locales et plus de } 10 \text { 000 adhérents à travers le } \\
\text { Maroc. }\end{array}$ \\
\hline $\begin{array}{l}\text { Association nationale des diplômés chômeurs du Maroc } \\
\text { (ANDCM) }\end{array}$ & $\begin{array}{l}\text { Crée en 1991, elle dispose d'une centaine de } \\
\text { sections locales. Se voulant «progressiste, } \\
\text { indépendante, populaire et démocratique », ses } \\
\text { protestations ont contribué à banaliser } \\
\text { l'occupation de la rue au Maroc. La section de } \\
\text { Casablanca est réactivée en 2010 et animée par } \\
\text { des militants d'extrême gauche (stalinistes, } \\
\text { maoïstes, etc.) }\end{array}$ \\
\hline $\begin{array}{l}\text { Association pour la taxation des transactions financières } \\
\text { et pour l'action citoyenne - Comité pour l'annulation } \\
\text { de la dette du Tiers Monde- Maroc (ATTAC-CADTM) }\end{array}$ & $\begin{array}{l}\text { Créée en 2000 au Maroc, cette organisation } \\
\text { altermondialiste fait partie d'un réseau } \\
\text { international regroupé sous forme de coordination } \\
\text { autour d'une plateforme commune. Depuis 2005, } \\
\text { elle connait une restructuration qui se traduit par } \\
\text { une forte présence du courant Almounadil-a } \\
\text { (trotskystes) au sein de ses instances. Elle compte } \\
\text { près de 500 adhérents. }\end{array}$ \\
\hline $\begin{array}{l}\text { Créé en 2009, d'abord en tant que groupe sur } \\
\text { Facebook, pour défendre la liberté de conscience, }\end{array}$ \\
\hline MALI)
\end{tabular}




\begin{tabular}{|l|l|}
\hline & $\begin{array}{l}\text { de culte, d'orientation sexuelle et plus } \\
\text { généralement l'instauration d'un État laïc. }\end{array}$ \\
\hline $\begin{array}{l}\text { Réseau des associations de quartier du Grand Casablanca } \\
\text { (RESAQ) }\end{array}$ & $\begin{array}{l}\text { Impulsé en 2003 autour d'une cinquantaine } \\
\text { d'associations de quartier, par un ancien détenu } \\
\text { d'extrême gauche, il vise à renforcer les capacités } \\
\text { des associations et à servir d'intermédiaire avec } \\
\text { des partenaires nationaux et internationaux. }\end{array}$ \\
\hline
\end{tabular}

Les «indépendants» méritent une attention particulière. Au sein du M20, cette autolabellisation est revendiquée par des cyberactivistes, des citoyens plus ou moins ordinaires, des acteurs de la nébuleuse associative ${ }^{30}$. Les frontières entre espace associatif, arène syndicale et scène politique n'étant pas étanches, nombreux sont les multipositionnés qui mettent en veille leur appartenance passée ou présente à une organisation politique pour exprimer à leur tour de la défiance à l'égard de la politique des « organisations ». Selon les propos des militants les plus aguerris, la principale ligne de partage oppose, d'une part, la «culture politique traditionnelle » fondée sur l'hégémonisme, la valorisation du leadership, de la hiérarchie et de la centralisation et, d'autre part, une culture inspirée par l'éducation populaire et les forums sociaux, privilégiant l'horizontalité, l'absence de leaders et de porte-parole, la décentralisation.

\section{Les « indépendants »}

Tout au long de l'histoire contemporaine marocaine, les registres de l' «indépendance », du « non organisé » ou du « non-partisan » servent d'énonciation à différents types de luttes politiques. À partir des années 1960, à la veille de chaque scrutin électoral, des relais de la monarchie s'approprient ce répertoire pour mobiliser contre les partis du Mouvement national ; ce qui ne les empêche pas, par la suite, de se constituer en partis dits administratifs. À la fin des années 1980, les associations de plaidoyer connaissent un nouvel élan et cherchent à recruter des « indépendants » en vue de rassembler au-delà du cercle des militants des organisations politiques ; mais les tensions internes ne tardent pas à se cristalliser autour d'une opposition entre « indépendants » et « partisans ». Peu à peu, l'énoncé « indépendant» finit par condenser le malaise diffus à l'égard d'un magma informe et à géométrie variable : la « «classe politique », « souillée », « corrompue », « divisée »» ${ }^{31}$, « makhzénisée »; le secteur associatif «clientélisé » à travers la redistribution particulariste de la manne financière émanant de l'Initiative nationale pour le développement humain (INDH) ; les organisations politiques en marge de la politique instituée. Inversement, il arrive que les opposants politiques se méfient des « indépendants » et les assimilent de potentiels « infiltrés ».

\section{La genèse du M20}

En dépit de l'intensité des interactions qui caractérisent cette construction sociale et historique qu'est le «monde arabe », la chute de Zine El Abidine Benali, le 14 janvier 2011, puis celle de Hosni Moubarak, le 11 février 2011, sont loin de produire un effet domino. Certes, des canaux tels qu'Al Jazeera favorisent l'amplification du sentiment d'appartenance à une même communauté imaginée et les réseaux sociaux contribuent à élargir l'éventail des voies de communication. Néanmoins, ils ne suffisent ni à déclencher mécaniquement une vague de protestation, ni à court-circuiter les autres médiations identifiées par les sociologues des mouvements sociaux ${ }^{32}$, ni à marginaliser les militants aguerris au profit de " nouveaux acteurs » (jeunes « dépolitisés », cyberactivistes). La description de la genèse du M20 permet d'identifier

\footnotetext{
${ }^{30}$ Il s'agit de membres d'organisations de plaidoyer, d'associations constituées autour d'une cause identitaire (les Amazighs) ou d'intérêts d'un secteur ou d'une catégorie de l'espace social (diplômés-chômeurs, habitants des bidonvilles, quartiers, etc.)

${ }^{31}$ Mounia Bennani-Chraïbi, « Jeux de miroir de la "politisation” : les acteurs associatifs de quartier à Casablanca », Critique internationale, 50, 2011, p. 55-71, dont p. 59.

${ }^{32}$ Dieter Rucht, «The Transnationalization of Social Movements. Trends, Causes, Problems », dans Donatella della Porta, Hanspeter Kriesi, Dieter Rucht (eds), Social Movements in a Globalizing World, Londres, Macmillan, 1999 , p. 223-244.
} 
les «processus vécus de diffusion $»^{33}$, la diversité des lieux de gestation du mouvement, la pluralité des canaux à travers lesquels se font les connexions entre différents individuels et collectifs, de même que les enchaînements et les interactions qui favorisent la mobilisation audelà du cercle des pionniers.

D'après les entretiens réalisés, la chute des présidents tunisien et égyptien à quelques semaines d'intervalle amène des acteurs marocains à calculer autrement. Tout d'abord, les grilles d'intelligibilité du jeu politique se brouillent, les horizons du possible et du faisable s'élargissent. D'après un membre d'ATTAC-Cadtm, âgé de 22 ans, « après la Tunisie, je me disais : imagine qu'une chose pareille se produise au Maroc. Mais après l'Égypte, je me suis dit: il faut absolument que quelque chose se produise au Maroc ». Dans un processus d' " attribution de similarité $»^{34}$, les interviewés établissent une identité entre les problèmes qui se posent en Tunisie, en Égypte, au Maroc : crise économique et sociale, chômage des diplômés du supérieur, discrédit de la "mascarade politique», monopolisation du pouvoir et des richesses par le roi et par « son premier cercle». À partir de là, ils anticipent des chances plus ou moins similaires de succès de la protestation ${ }^{35}$. Ne serait-ce que dans un premier temps, les pionniers du M20 attribuent les succès tunisiens et égyptiens à quatre facteurs principaux : la mise en avant de "jeunes dépolitisés », l'absence de leadership, de hiérarchie et de centralisation, l'importance de la spontanéité et de l'innovation et, surtout, la dissolution des identités (organisationnelles, idéologiques, ethniques, etc.). C'est à partir de ce cadrage que les initiateurs du M20 construisent l'image d'un mouvement « jeune » qui va au-delà des clivages idéologiques et entérinent une inversion des rôles entre militants aguerris et nouveaux entrants, de manière à faire apparaître les leaders d'antan comme de simples suiveurs.

Si les médias et les réseaux sociaux jouent un rôle primordial dans les processus d'identification, ils ne suffisent pas à faire descendre les protestataires dans la rue le 20 février. En effet, c'est dans la jonction entre une pluralité de réseaux et de lieux que se font les connexions entre cyberactivistes ${ }^{36}$ (et parmi eux ceux qui militent dans des structures organisées), militants associatifs, acteurs multipositionnés.

Pendant les derniers jours de l'année 2010, les acteurs de l'espace protestataire marocain suivent avec attention les mobilisations qui se produisent chez leurs voisins. Les autorités sont à l'affût. Le 13 janvier 2011, la Coordination marocaine de soutien aux démocrates tunisiens $(\text { CoMaSoDeT })^{37}$ organise un sit-in devant l'ambassade de Tunisie, violemment dispersé. Pourtant les actions de solidarité avec la Palestine, l'Irak ou le Liban sont quasi routinières au Maroc. À partir de la chute de Ben Ali, les manifestations qui fêtent la révolution tunisienne sont toutefois tolérées. Dans le même mouvement, des communiqués et des déclarations sont publiés par des associations, des syndicats et des partis, dans la presse écrite et sur leurs sites web, d'abord pour soutenir le peuple tunisien et ensuite pour le féliciter.

Pendant ce temps, la fièvre gagne les Facebookers marocains et la médiatisation de l'impact révolutionnaire de ce réseau social suscite la curiosité des néophytes. Dès le 14 janvier, trois jeunes cyberactivistes originaires de Meknès créent sur Facebook le groupe «Des Marocains dialoguent avec le Roi ». H. A. est l'un d'entre eux. Jusque-là, cet étudiant en

\footnotetext{
33 Isabelle Sommier, «Diffusion et circulation des mouvements sociaux», dans Olivier Fillieule, Éric Agrikoliansky, Isabelle Sommier (dir.), Penser les mouvements sociaux, La Découverte, 2010, p. 101-120, dont p. 115.

${ }^{34}$ D. Strang, J. W. Meyer, « Institutional conditions for diffusion », art. cité.

${ }^{35}$ Sur les notions de «jeux d'assurance » et de «bandwagon effect», processus qui conduisentt les suiveurs à participer à un mouvement après avoir observé et évalué ses chances de succès, voir : Dennis Chong, Collective Action and the Civil Rights Movement, Chicago, The University Chicago Press, 1991.

${ }^{36}$ Voir Driss Ksikes, « Genèse du cyber-activisme au Maroc », Economia, Cesem, Rabat, 12, 2011, p. 80-83.

${ }^{37}$ Créée en 2005 en solidarité avec les militants tunisiens en grève de la faim, elle rassemble plus d'une vingtaine d'associations, des syndicats, des partis - notamment de la gauche radicale (RGD) mais également la jeunesse de l'USFP -, le Groupe national de soutien à l'Irak et à la Palestine.
} 
ingénierie, âgé de 22 ans, n'a jamais adhéré à quelque organisation que ce soit et son père, un enseignant, évoque peu ses sympathies de jeunesse pour l'extrême gauche. C'est dans les groupes de discussion « progressistes » sur internet qu'il débat de la chose publique depuis trois ans $^{38}$. Le 25 janvier, il regarde Al Jazeera au café avec un ami et ressent une énorme frustration. Tous les soirs, il furète sur la toile espérant dénicher un appel à manifester lancé par l'une ou l'autre des organisations de la gauche radicale. Exaspéré, il décide de passer à l'action avec ses deux amis. Le 27 janvier, les trois jeunes rebaptisent leur groupe « Mouvement liberté et démocratie maintenant » et lancent un appel à manifester le 27 février, dans les grandes places publiques, devant les sites des préfectures et des gouvernorats. Le groupe compte 3000 membres le 27 janvier, 6000 membres le 3 février. L'appel comporte six revendications à vocation fédératrice allant de l'abrogation de la constitution et la " désignation d'une commission constituante parmi des personnalités les plus qualifiées et les plus intègres, chargée de rédiger une nouvelle constitution qui donne à la monarchie sa taille naturelle » à la création d'une caisse d'indemnisation du chômage. D'après H. A., l'option implicite pour une monarchie parlementaire relève d'un choix « rationnel », d'un projet « réalisable » en affinité avec le rejet par les signataires de la violence, de l' « anarchie » et du « blanquisme $»{ }^{39}$. Le choix de la date prête le flanc aux attaques des autorités : le 27 février coïncide avec l'anniversaire de la proclamation en 1976 de la République arabe sahraouie démocratique par le front Polisario. Dès lors, la date est avancée au 20 février.

Le 28 janvier, le Jour de la colère en Égypte s'accompagne de fébrilité dans le réseau internet marocain. Un ex-affilié de la jeunesse de l'USFP de Salé lance une vidéo sur YouTube, où il lit l'appel du Mouvement liberté et démocratie maintenant. Désormais, l'appel du 20 février est incarné, il devient une référence pour les groupes qui se multiplient sur Facebook, faisant des émules d'une région à l'autre du Maroc.

Le 3 février, Al Massae, le quotidien arabophone le plus lu au Maroc, attaque les jeunes qui ont lancé l'appel et les accuse de collusion avec les Algériens et le Polisario. La campagne de disqualification animée par des ministres et des médias officiels provoque un mouvement de solidarité avec « les jeunes », tout en médiatisant la date du 20 février, devenue l'événement politique du moment.

À Rabat, les manifestations de soutien au peuple égyptien offrent l'occasion à un groupe de jeunes de se réunir régulièrement. Pour la plupart, ils sont enfants de militants, sympathisants ou membres des réseaux de gauche. Dès le départ, le soutien de l'AMDH est fondamental. Forte de son rôle pionnier au sein de plusieurs réseaux, elle constitue à la fois une courroie de transmission et un réservoir en ressources humaines et logistiques; elle contribue par ailleurs à la socialisation et au renouvellement générationnel de la gauche radicale. Ce n'est donc pas surprenant que son siège devienne le QG des jeunes de Rabat. C'est là qu'ils réalisent la vidéo appelant à « sortir » le 20 février et postée sur YouTube le 12 février. À visage découvert, ils commencent par s'identifier sans révéler leur affiliation : «je suis marocain », «je suis marocaine $»$.

La diffusion de cette vidéo donne des visages au mouvement, qui cesse d'être une rumeur virtuelle. La campagne de dénigrement et d'intimidation se précise : les membres du M20 sont assimilés à des «traitres » qui remettent en cause les fondements de la nation (Dieu, la patrie, le roi), des « marginaux » qui transgressent ses valeurs (des convertis au christianisme, des « athées », des « rompeurs du jeûne », des « homosexuels »). Les « services » commencent à agir : des coups de téléphone anonymes, des visites plus ou moins «courtoises» et des pressions sur les familles.

\footnotetext{
${ }^{38}$ Entretien, 18 décembre 2011.

${ }^{39}$ Alors même qu'il affirme ne pas avoir été imprégné par l'expérience politique de son père, il recourt au vocabulaire des générations militantes précédentes.
} 
À Rabat, les rassemblements de solidarité avec la révolution égyptienne sont presque quotidiens et désormais tolérés. Ils favorisent les échanges entre jeunes et moins jeunes, militants de gauche et islamistes d'Al Adl Wal Ihsane, à tel point que le sit-in du samedi 12 février devant le parlement se transforme en un forum de débat où un objectif commun s'impose : « descendre dans la rue et marcher ensemble, le 20, partout au Maroc ».

Ce samedi même, le groupe de jeunes de Rabat se réunit au siège de l'AMDH pour réécrire la plateforme du M20, ajouter de nouvelles revendications, en puisant cette fois-ci dans le vocabulaire politique de l'opposition de gauche ${ }^{40}$. La monarchie n'est plus mentionnée et le seuil politique privilégié est désormais : « une constitution démocratique représentant la vraie volonté du peuple rédigée par une assemblée constituante élue ».

Pendant ce temps, à Casablanca, une rencontre réunit les représentants nationaux des jeunesses des partis de la gauche radicale (RGD). Ils publient la première déclaration de soutien à l'appel du 20 février, émanant d'organisations politiques. Le lendemain, leurs positions sont reprises par les instances nationales de leurs partis, le 14 février par dix-sept associations de droits humains ${ }^{41}$. Entre temps, les Organisations et démocrates marocains lancent l'Appel de Dakar pendant le Forum social mondial (6-12 février) où ils ont l'occasion d'échanger avec des Tunisiens, des Égyptiens et des ressortissants d'autres pays frères en effervescence ${ }^{42}$.

Les organisations islamistes ne tardent pas à prendre le relais : la jeunesse d'Al Adl déclare son intention de participer aux manifestations du M20 le 16 février ; celle du PJD publie une déclaration de soutien le 17, puis la retire suite aux pressions exercées par sa hiérarchie en pourparlers avec les autorités ${ }^{43}$, mais des jeunes de ce parti finissent par se rallier à un parlementaire du PJD pour constituer le groupe Baraka ${ }^{44}$. Désespérés par la réserve de leur leadership à l'égard du M20, des membres de l'USFP publient le 18 une déclaration signée par les «Usfpistes du 20 février ». Après son scepticisme de départ, un parlementaire Usfpiste se rallie publiquement; il a le sentiment que le mouvement fait l'objet d'une " attaque concertée » de ministres, de partis, de l'agence de presse officielle : «Quand ils prennent ces positions, cela veut dire pour moi que je dois être de l'autre côté $»^{45}$.

Impulsé par la marge, le mouvement de protestation s'étend rapidement, faisant émerger une configuration d'alliance qui semble bousculer les barrières politiques et sociales qui se dressent en phase routinière. La gauche radicale et non gouvernementale s'engage au sein du mouvement dès la première heure, suivie par les islamistes d'Al Adl. La frontière entre politique instituée et espace protestataire se brouille : la principale force au sein de l'opposition parlementaire (les islamistes du PJD) et les partis de la gauche gouvernementale (USFP, PPS) sont secoués de l'intérieur. La médiatisation de figures inconnues jusque-là amplifie la diffusion de l'image d'un mouvement « jeune » qui va au-delà des clivages idéologiques. La pluralité des revendications des plateformes du M20, leur caractère flou et non hiérarchisé renforcent la dimension fédératrice du mouvement et favorisent la jonction avec une multiplicité d'entrepreneurs de cause, porteurs de demandes sociales et politiques plus ou moins

\footnotetext{
${ }^{40}$ Depuis l'indépendance, la question constitutionnelle constitue un enjeu pour des générations d'opposants. Voir Jean-Noël Ferrié, Baudouin Dupret, « La nouvelle architecture constitutionnelle et les trois désamorçages de la vie politique marocaine », Confluences Méditerranée, 2011, 78, p. 25-34.

${ }^{41}$ Créé entre le 20 et le 23 février, le Conseil national d'appui au M20 (CNAM20) regroupe des organisations politiques, de même que de grandes centrales syndicales et une centaine d'associations.

${ }^{42}$ Le mois de février 2011 voit des appels à manifester le 6 en Syrie, le 11 au Yémen, le 12 en Algérie, le 14 à Bahreïn et en Iran, le 17 en Libye.

${ }^{43}$ Le 17 février, le secrétaire général du PJD annonce le boycott du M20 par son parti. Dès le lendemain, le procureur relâche un membre du secrétariat général du PJD, arrêté pour corruption. Bien davantage, le 21 février ce dernier est nommé au Conseil économique et social (CES).

${ }^{44}$ Selon son fondateur, ce synonyme de Kifaya (« ça suffit ») fait référence au mot d'ordre lancé en 2004, en Egypte, par le mouvement du même nom.

${ }^{45}$ Entretien, juillet 2011.
} 
préexistantes, des plus « universelles » aux plus sectorielles. Le soutien public de quelques intellectuels, artistes, journalistes et hommes d'affaires contribue à dégager une impression de diversité sociale. Dans ce processus de diffusion, transparaissent de manière intriquée des « voies de diffusion non relationnelles», des médiations informelles, des relais organisationnels, des réseaux plus ou moins « dormants ${ }^{46}$.

Tout au long de la dynamique qui précède le 20 février, les autorités envoient des signaux ambivalents qui accentuent, chez les pionniers et les suiveurs potentiels du M20, le sentiment qu'une brèche est ouverte. En effet, dès la chute de Ben Ali, différentes mesures sont prises : accélération des négociations avec les diplômés chômeurs et avec les syndicats en vue d'interrompre les protestations sectorielles antérieures, doublement du budget de la caisse de compensation, recommandation aux prédicateurs du vendredi de mettre en garde contre le chaos, etc. D'une part, les autorités essaient d'anticiper, de gagner du temps, de disqualifier les jeunes qui appellent à manifester le 20 février et de décourager l'établissement de jonctions entre les jeunes de Facebook, les acteurs de la scène politique instituée, les syndicats, les islamistes, et les mouvements des diplômés chômeurs. D'autre part, elles se mobilisent pour véhiculer l'idée de «l'exception marocaine »; des annonces rassurantes laissent entendre qu'il n'y aura pas de répression le 20 février : à l'inverse de ses voisins, le Maroc serait un pays démocratique où les mouvements sociaux seraient routiniers.

\section{La genèse de la coordination du M20 à Casablanca}

À Casablanca, dès le départ, l'initiative est prise par des acteurs « organisés ». Dans l'effervescence de ce mois de février, l'embryon du M20 Casablanca se constitue à partir de la jonction entre deux réseaux sociopolitiques structurants.

Le premier correspond à la nébuleuse de gauche dans toutes ses gradations. L'un de ses noyaux est l'Espace Casablanca pour le dialogue de la gauche (ECDG), une plateforme initiée en avril 2008 par des militants multipositionnés, plutôt en marge de leurs partis (le PSU et l'USFP). Après les échecs électoraux des partis de la gauche gouvernementale et radicale de 2007, leur objectif est de constituer un lieu de réflexion en vue d'unifier la gauche. C'est l'ECDG qui appelle à la rencontre de l'Espoir, le 12 février, dans le siège du PSU, situé au centre-ville, suffisamment accueillant pour abriter les réunions de toutes sortes d'acteurs associatifs et protestataires. La plupart des présents se connaissent déjà, ayant partagé des expériences associatives (Tableau 2). Certains se sont côtoyés au cours des cinq dernières années dans le cadre de la Coordination contre la cherté de la vie jusqu'en 2009, puis dans les Comités de jeunes pour la libération des détenus politiques (2008-2009) et dans le Comité de l'habitat mobilisé jusqu'en janvier 2011 auprès des bidonvillois.

Le deuxième pôle structurant, celui d'Al Adl Wal Ihsane, est plus fermé. En effet, l'entrée au sein de cette organisation est encadrée par des règles strictes; sa discipline militante constitue en soi un filtre sélectif ${ }^{47}$. Un programme éducatif individuel et collectif régit la vie spirituelle et mondaine des adhérents, qui sont par ailleurs incités à se surpasser dans toutes leurs sphères de vie, à se former sur les plans intellectuel et sportif, à consentir des efforts matériels au bénéfice de l'organisation. Très présente au sein des campus, notamment depuis la fin des années 1980, cette organisation politique non autorisée assure son rayonnement audelà du cercle des fidèles en développant un tissu associatif, des activités de bénévolat, des réseaux sociaux sur internet.

\footnotetext{
${ }^{46}$ Voir à ce sujet Verta Taylor, « La continuité des mouvements sociaux. La mise en veille du mouvement des femmes », dans Olivier Filleule (dir.), Le désengagement militant, Paris, Belin, 2005, p. 229-250.

${ }^{47}$ Voir notamment Mohamed Darif, al-Islamiyyun al-maghariba : hisabat as-siyyasa fi al-'amal al-islami 19691999 [Les islamistes marocains : les calculs politiques dans l'action islamiste 1969-1999], Casablanca, Al-Majalla al-maghribiyya li 'ilm al-ijtima' as-siyyasi, 1999, p. 67-76.
} 
Les frontières ne sont pas totalement étanches entre ces deux réseaux qui s'enracinent dans le même univers citadin des éduqués, plus ou moins jeunes, issus des catégories populaires ou de la classe moyenne. Les lignes de partage les plus visibles transparaissent autour de questions politiques (laïcité versus État islamique), morales et religieuses (degré de respect des prescriptions religieuses, degré de promotion des valeurs individuelles, etc.). Les stigmatisations mutuelles sont véhiculées par les médias et les écrits politiques, ou à travers des expériences directes dans le campus, dans le quartier. Il existe une mémoire de la confrontation, y compris à l'occasion des marches de solidarité avec la Palestine pendant lesquelles il arrive que gens de gauche et islamistes s'arrachent les micros pour scander «Palestine arabe » ou «Palestine islamique ${ }^{48}$. Toutefois, le sentiment de proximité n'est pas exclu entre ceux qui rejettent la politique instituée et l'hégémonie monarchique, qu'ils soient d'extrême gauche ou islamistes. Enfin, signalons les cas des familles composites politiquement ou des jeunes socialisés dans un milieu islamiste qui deviennent « gauchistes », qui ne sont pas exceptionnels dans notre échantillon.

Étant donné le caractère fortement centralisé et hiérarchisé d'Al Adl, la participation de sa jeunesse au M20 est décidée en haut lieu. Et ce n'est qu'à la suite de la publicisation de cette décision, le 16 février, que les pionniers du milieu de gauche à Casablanca établissent le contact avec les responsables de la jeunesse d'Al Adl à l'échelle locale. Cependant, le profil de ceux qui assurent concrètement la connexion n'est pas anodin. En lien avec leur idéologie, leurs expériences passées et leurs dispositions personnelles, deux trotskystes d'ATTAC-Cadtm Casablanca jouent un rôle prééminent dans la jonction et dans tout le travail qui suivra pour la maintenir. Après avoir milité au sein de groupuscules estudiantins, ils s'investissent dans les dynamiques protestataires que connaît Casablanca au cours des dernières années. D'après eux, révolutionner la société nécessite l'évitement du conflit avec les autres forces sociales et politiques. Adeptes du slogan «marcher séparément et frapper ensemble », ils défendent déjà l'idée d'intégrer les Adlistes dans la Coordination contre la cherté de la vie, mais les autres composantes de gauche s'y opposent farouchement. L'un d'entre eux revient du Caire où il séjourne dans le cadre d'une formation Cadtm jusqu'à 30 janvier 2011. Il a vécu l'expérience de la place Tahrir, observé l'entraide entre révolutionnaires égyptiens de toutes tendances. Pendant les mois à venir, les deux militants sont perçus comme fédérateurs et appréciés pour leur abnégation et leur caractère conciliant. Comme les autres chevilles ouvrières du M20, ils sont plus ou moins disponibles biographiquement (l'un est au chômage, l'autre est enseignant).

Très vite, les pionniers du M20-Casablanca mettent en place des dispositifs visant à organiser une action commune dans le cadre d'une coalition hétéroclite. Imprégnés par les expérimentations et les échecs du passé autant que par ce qu'ils retiennent du «modèle » de la place Tahrir, ils anticipent et tentent de se donner les moyens de résister aussi bien à la répression qu'à la cooptation par le régime. Leurs mots d'ordre plus ou moins explicites sont : fédérer, invisibiliser les identités particulières ${ }^{49}$, éviter l'hégémonisme ou le détournement de l'action par une composante politique en particulier, décourager toute vocation au leadership individuel ou collectif. L'accent est également mis sur la nécessité de s'ouvrir suffisamment pour rallier de nouveaux entrants et favoriser l'innovation.

Entre le 15 et le 18 février, les réunions préparatoires puisent dans les savoir-faire estudiantins en instaurant deux règles : toute intégration à un comité doit être validée par l'AG ; la seule instance décisionnelle officielle du mouvement est l'AG. Les participants à l'AG du 18 février signent une liste de présence : dans la colonne réservée à l'affiliation, tous s'identifient en tant que membres du M20. Au cours des AG qui se déroulent pendant les mois suivants,

\footnotetext{
${ }^{48}$ Mounia Bennani-Chraïbi, « Les conflits du Moyen-Orient au miroir des communautés imaginées : la rue arabe existe-t-elle ? Cas du Maroc », A Contrario, 5(2), 2008, p. 147-156.

${ }^{49}$ Sur les modes de présentation de soi en politique, voir Annie Collovald, «Identité(s) stratégique(s) », Actes de la recherche en sciences sociales, 73, 1988, p. 29-40.
} 
toute personne qui cite le nom d'une organisation est rappelée à l'ordre. Par ailleurs, lors de la constitution des premiers comités (logistique, slogans, mobilisation, communication), le choix des coordinateurs (des membres d'ATTAC, de l'ANDCM, de MALI), approuvé par l'AG, semble obéir à des critères implicites : privilégier des personnes de confiance, éviter à la fois les acteurs non organisés et les membres des partis, de manière à mettre en avant des personnes dotées de compétences militantes tout en garantissant l'indépendance de la coordination à l'égard des partis. Les membres des organisations politiques sont toutefois incités à adhérer au comité de la logistique, pour le faire bénéficier de leur carnet d'adresses et mobiliser les soutiens matériels nécessaires à l'impression des tracts, à la réalisation de banderoles, à l'accès à un dispositif de sonorisation.

Avant le dimanche 20 février, l'absence des « jeunes de Facebook » inquiète les pionniers du M20 à Casablanca. Imprégnés par le modèle des internautes révolutionnaires tunisiens et égyptiens, ils décident de diffuser les conclusions de l'AG du 18 février sur FB et d'inviter les jeunes Facebookers à se joindre à eux le dimanche 20 février à 10 heures, sur la Place Lahmam. La centralité de cette place située dans le cœur administratif de Casablanca, son caractère spacieux et sa proximité de la préfecture en font l'un des lieux privilégiés des sit-in qui se déroulent dans la capitale économique. Dans le tract diffusé, l'action n'est pas « nommée » : il n'est question ni de «sit-in» (weqfa), ni de «marche» (masira). D'après un militant d'ATTAC, lancer un appel sur Facebook laissait présager la participation de personnes « différentes »; il fallait donc « laisser la liberté » aux nouveaux venus de « faire quelque chose de différent $\gg{ }^{50}$.

Le 20 février est vécu comme un succès par les pionniers du M20 à Casablanca pour trois raisons principales : le nombre des participants, leur diversité, l'atmosphère qui prévaut jusqu'à l'appel à la dispersion lancé par les organisateurs vers 16 heures. En effet, au pic de la mobilisation, la place réunit près de 6000 personnes selon la presse. De grands entrepreneurs, des artistes célèbres, des acteurs associatifs, d'anciens détenus et même quelques parlementaires se font remarquer. Des jeunes qui n'ont jamais pris part à aucune élection, à aucune action protestataire, qui n'ont jamais adhéré à aucune organisation ont répondu à l'appel ; ils sont venus en famille, avec des voisins, ou encore avec leur "bande de copains ». Les préparatifs des jours précédents et le travail de négociation mené in situ par les membres des comités du M20 favorisent "l'unification » de l'action : à quelques exceptions près, "les slogans et les banderoles se sont unifiées, les identités se sont dissoutes comme en Tunisie et à place Tahrir $[\ldots]$, l'atmosphère est bon enfant $»^{51}$.

Toutefois, suite à l'appel à la dispersion, des personnes proviennent de la Médina, un quartier populaire limitrophe, après la fin d'un match de football. Ils tentent de prolonger l'événement par une marche dans une ambiance de sortie de stade, "chaotique " selon les militants chevronnés qui les observent. Des jeunes, présents depuis 10 heures, appellent alors au sit-in illimité ( $i$ 'tisam) ; ils ont ramené des tentes dans l'espoir de reconstituer la place Tahrir au centre de Casablanca. Les membres du M20 qui ne se sont pas encore dispersés improvisent un cordon de sécurité, mais se sentent dépassés. Lorsque l'action organisée par le M20 prend fin à $16 \mathrm{~h}$, ce sont les forces de sécurité, discrètes jusque-là, qui interviennent de manière autolimitée ; l'évacuation ne s'achève qu'après 22 heures.

Après ce dimanche, le M20 s'inscrit dans la durée sur les plans national et local, avec des phases de flux et de reflux. Dans un «jeu d'échelles » entre l'interne, le local, le national, le régional et l'international, un entrelacement d'actions, d'interactions et d'événements contribue - synchroniquement et diachroniquement - autant à la consolidation qu'à la reconfiguration de la coalition du M20, autant à l'extension du mouvement qu'à son essoufflement. Mais, pour en faciliter la lisibilité, nous dissocierons la présentation de ces deux processus si intriqués.

\footnotetext{
${ }^{50}$ Entretien, septembre 2011.

${ }^{51}$ Entretien avec un militant d'ATTAC, novembre 2011.
} 


\section{Le processus d'enracinement de la coalition du M20 et d'extension de la protestation}

Alors même que les germes de la dissension ne tardent pas à transparaître, une conjonction d'éléments favorise la consolidation du M20 à Casablanca et la poursuite de l'extension de la protestation : certains sont liés aux interactions avec les autorités et à la perception de ce qui se joue aux échelles nationale, régionale et internationale; d'autres à la dynamique interne au sein de la coordination.

\section{Des interactions qui amplifient la fluidité}

Après le 20 février, les autorités continuent à diffuser le message de "l'exception marocaine $»$ : exceptés quelques troubles jugés marginaux, les actions protestataires se sont déroulées dans un climat pacifique, attestant de la «maturité » du pays et de son caractère « démocratique ».

Le discours royal du 9 mars 2011 est à la fois perçu comme une reconnaissance du M20 et comme une tentative de lui couper l'herbe sous les pieds en présentant une offre de réforme susceptible de séduire. En effet, il annonce l'accélération de « la dynamique réformatrice » et $\mathrm{du}$ processus de régionalisation, une "réforme constitutionnelle globale », un référendum constitutionnel, etc. Il est précédé et suivi par la mise en place d'un dispositif institutionnel d'ouverture : Conseil économique et social (CES) le 21 février; transformation du Conseil consultatif des droits de l'homme (CCDH) en Conseil national des droits de l'homme (CNDH) le 4 mars avec la nomination comme secrétaire général d'un ancien détenu de la gauche non gouvernementale et ancien président du Forum marocain vérité et justice; création de la Commission consultative pour la révision de la constitution (CCRC) le 10 mars, de l'institution du « Médiateur» le 17 mars, etc. Les réactions internationales ne tardent pas : le roi est cité en exemple par les puissances occidentales et l'Union européenne exprime sa satisfaction par une augmentation tangible de l'aide apportée annuellement au Maroc.

Pendant cette séquence, le discours médiatique et politique dominant adopte un ton enthousiaste : «le M20, ce sont nos enfants», «nous sommes tous des M20», « le M20 a arraché ce que des années de lutte politique n'ont pas permis de réaliser ». À l'intersection entre espace protestataire et scène politique instituée, la dynamique du M20 continue à s'étendre dans un ensemble d'arènes allant des médias officiels aux conseils communaux. La gauche gouvernementale connaît une exacerbation des tensions internes entre «ceux qui ont pris l'habitude de n'avoir pour vis-à-vis que la monarchie » et ceux qui aspirent à la rupture avec la logique cooptative qui, d'après eux, a conduit au blocage de la politique instituée ; ces conflits s'expriment dans les médias classiques, mais surtout dans les échanges sur Facebook où le ton trahit l'effacement de la frontière entre énoncés réservés aux coulisses et discours publics. Le discours royal va au-delà des attentes des premiers, sans pour autant satisfaire les seconds. Quant à la gauche non gouvernementale qui soutient le M20, et plus précisément le PSU, c'est le refus de jouer un rôle de médiation entre le mouvement et les autorités qui l'emporte au sein d'une direction qui « se laisse entraîner par le M20 $»^{52}$.

Le mouvement est alors confronté à deux défis : comment répondre au discours royal sur le plan idéologique et sur le terrain ? Comment imposer la poursuite du mouvement ? À Casablanca, la répression du 13 mars permet à la coordination de sortir de ce dilemme. La confrontation avec les forces de sécurité s'étend jusqu'à l'entrée du siège du PSU où se tient le Conseil national du parti. Les leaders du PSU se joignent au M20 qui proclame « un sit-in

\footnotetext{
${ }^{52}$ Entretien avec un membre du PSU, décembre 2011.
} 
illimité » jusqu'à la libération de la centaine de militants arrêtés. Cet épisode est perçu par les vingt-févriéristes comme un message des autorités : le discours royal du 9 mars est un signal de clôture ; les revendications doivent désormais s'exprimer au sein des dispositifs de réforme annoncés. Suite à la médiatisation de l'événement, des organisations internationales publient des communiqués de soutien au M20.

Sur un autre plan, ce moment intense génère un sentiment de cohésion au sein du groupe. Pour les uns et les autres, " le gauchiste a pris le coup à la place de l'islamiste », " on a mis en pratique la leçon donnée par les Égyptiens à place Tahrir $\|^{53}$. Avant cette date, les Adlistes n'apparaissent pas au-devant de la scène. Les militants d'ATTAC continuent à jouer leur rôle fédérateur. C'est d'ailleurs l'un des leurs qui aurait proposé qu'un Adliste prononce le discours de clôture du sit-in du 6 mars et appelle à celui du 8 mars, organisé à l'occasion de la Journée internationale de la femme, dans l'espoir de lever les inquiétudes des sécularistes. À ce stade, aucune composante ne s'aventure à prendre la responsabilité d'un éventuel échec. En outre, la réussite est plus que jamais associée au fait de masquer les identités particulières.

\section{Lorsque le M20 gagne la « bataille de l'opinion " : 20 mars - 24 avril}

Si dans la séquence précédente, monarchie et M20 semblent ex aequo dans leur « bataille pour l'opinion $»^{54}$, les marches nationales ${ }^{55}$ du 20 mars et du 24 avril sont une consécration pour le mouvement. À l'échelle du Maroc, plus de cent coordinations organisent des actions protestataires le 24 avril. Pendant plus d'un mois, le mouvement donne le sentiment d'avoir « gagné » le Makhzen.

À Casablanca, la coordination du M20 se mobilise intensément pour arracher le droit de manifester sans demander d'autorisation ${ }^{56}$, pour démontrer sa capacité à faire nombre, à tenir la rue et les rangs. En effet, à partir du 20 mars, elle appelle à des " marches populaires » (masira sha 'biyya) quasi hebdomadaires, tandis que d'autres coordinations du M20 continuent à organiser des sit-in. Le summum de la discipline manifestante du M20-Casablanca est atteint le dimanche 24 avril, pendant une marche qui aurait rassemblé entre 10000 et 35000 manifestants (Photographie 1). Ce jour-là, le collectif manifestant est plus diversifié que jamais : il comporte non seulement les pionniers et les suiveurs parmi les membres des organisations qui soutiennent le M20, mais également des figures de la gauche gouvernementale, les membres du mouvement Baraka conduit par un parlementaire du PJD, des hommes d'affaires et des artistes. Les porteurs de demandes sectorielles sont particulièrement présents : des vendeurs ambulants, des bidonvillois condamnés à l'expulsion, des retraités des Forces auxiliaires, des commerçants des fruits et légumes regroupés derrière une banderole revendiquant que le directeur du marché de gros « dégage », etc.

\section{Photographie 1 postée sur Facebook par le M20- Casablanca}

Marche du 24 avril 2011 au centre-ville de Casablanca. Un dispositif permet d'unifier la marche avec au moins un membre du service d'ordre toutes les cinq rangées, un mégaphone toutes les dix rangées, une grande banderole du M20 (6 mètres sur 1,20 mètre) tous les 600 mètres, quatre véhicules sonorisés intercalés à chaque kilomètre.

\footnotetext{
${ }^{53}$ Entretien avec un Adliste, avril 2011.

54 « Gagner » ou « perdre » la « bataille de l'opinion » fait partie du vocabulaire employé par nos interviewés.

55 Les actions protestataires mensuelles ont un caractère national, les autres relèvent de l'initiative des coordinations.

${ }^{56}$ La manifestation est régie par le Dahir des libertés publiques et obéit en principe au régime déclaratif.
} 


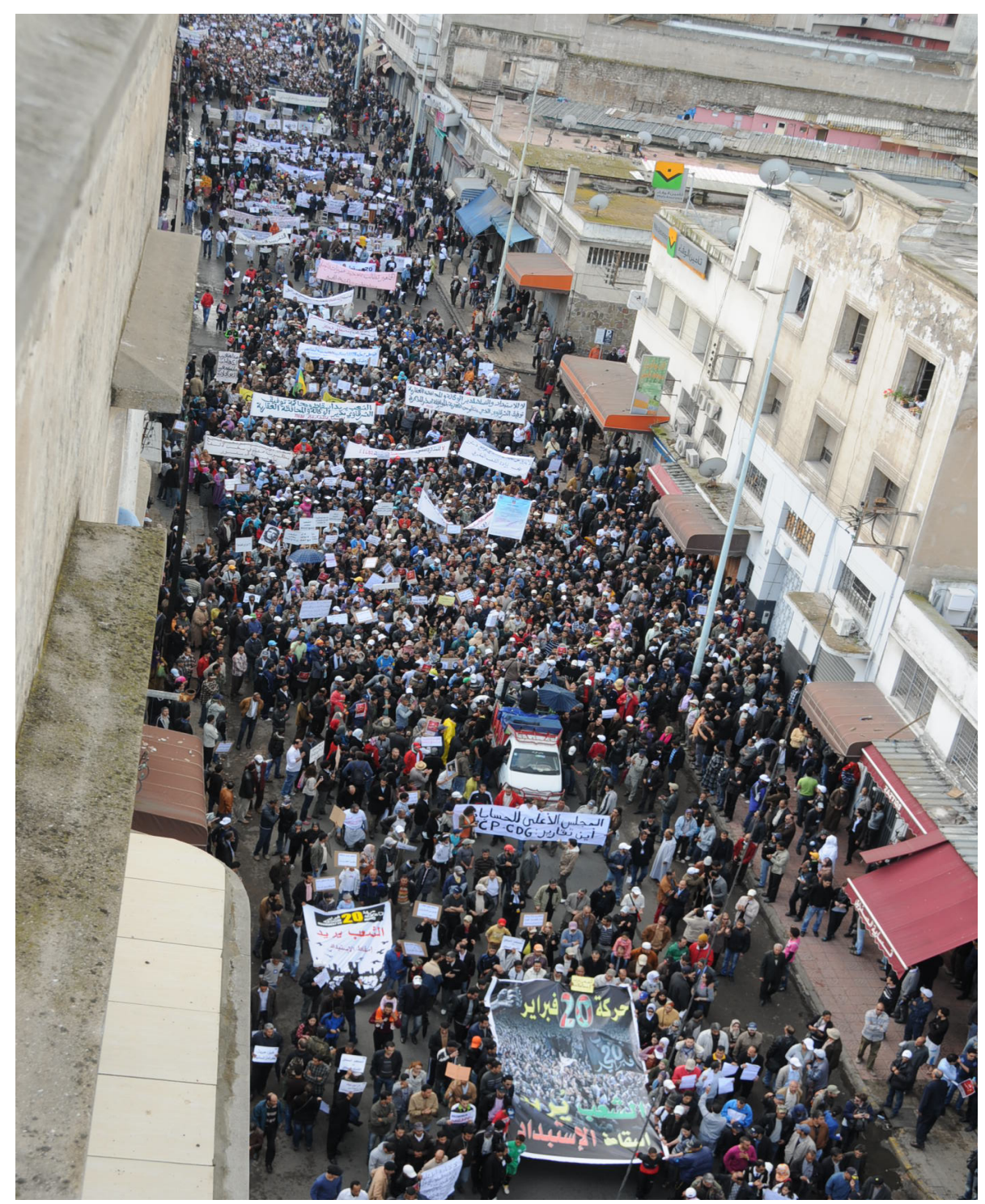

Pendant la séquence allant du 20 mars au 24 avril, les forces de sécurité se tiennent à distance des cortèges manifestants. Les acteurs en présence ont conscience que les autorités veulent faire bonne figure et éviter autant que possible le recours à la répression à la veille d'un rendez-vous important. En effet, la réunion du Conseil de sécurité doit se prononcer avant fin avril au sujet de la prorogation du mandat de la Mission des Nations Unies pour l'organisation d'un référendum au Sahara occidental (MINURSO). Pendant ce temps, les autorités poursuivent leurs tentatives d'achat de la paix sociale et 190 détenus sont libérés le 14 avril ${ }^{57}$.

\section{Une dynamique interne surgénératrice d'engagement}

Au cours des deux séquences (de fin février au 24 avril), les membres du M20 les plus investis dans l'organisation de la protestation croient en la capacité du mouvement à exercer une pression sur le Makhzen. Les batailles menées sont interprétées comme des succès et tendent à souder le collectif. Nombreux sont ceux qui ont le sentiment de vivre une occasion historique à ne pas rater. L'entente entre Adlistes et militants de sensibilité de gauche est à son

\footnotetext{
${ }^{57}$ Parmi eux, des militants sahraouis et des personnes arrêtées suite aux attentats du 16 mai 2003 à Casablanca, dont des membres présumés de la « Salafiyya jihadiyya ».
} 
apogée. Les filles en décolleté et en jeans moulants fraternisent avec les voilées. Une observatrice constate que des Adlistes consentent désormais à serrer la main des filles ${ }^{58}$.

Par ailleurs, le calendrier dense des activités militantes produit un « effet surgénérateur de l'engagement ${ }^{59}$ : marches hebdomadaires, AG, réunions des comités, campagnes de distribution des tracts, actions ponctuelles pour dénoncer telle administration ou se mobiliser pour la libération de militants arrêtés. Sans compter que l'atmosphère d'effervescence des marches crée une quasi-addiction.

Enfin, la vitalité de la coordination du M20 est tributaire des sociabilités réactivées ou nouvelles qui se tissent, par groupe affinitaire notamment, autour des sièges des organisations qui soutiennent le M20, dans les locaux eux-mêmes ou dans les cafés du centre-ville. Sous l'égide des plus expérimentés, les néophytes - jeunes et moins jeunes - se socialisent à de nouveaux univers de sens et de pratiques (militants, artistiques, etc.). Parmi eux certains font le pas d'adhérer à l'une des organisations qui soutiennent le M20, d'autres ont le sentiment d'appartenir à une nouvelle famille, d'autres encore vivent des histoires d'amour ; lorsque l'une d'entre elles se concrétise par une cérémonie de mariage, les slogans vingt-févriéristes accompagnent les youyous.

\section{Le processus de reconfiguration et de désagrégation de la coalition du M20}

Les phases de reconfiguration et d'affaiblissement de la coalition du M20 ne succèdent pas mécaniquement aux séquences d'enracinement de la coordination et d'extension de la protestation. Dans le faisceau des occurrences perçues comme décisives ou des microévénements à peine perceptibles, il arrive que des interactions favorisent à court terme le déploiement du mouvement, en même temps qu'elles charrient dans leur sillage les germes mêmes qui contribuent à sa désagrégation. Cependant, si nous nous en tenons à une séquentialisation en lien avec les principales défections que connaît le M20 en 2011, nous pourrons retenir deux points d'inflexion. Suite à la marche du 24 avril, le mouvement enregistre des retraits, plus ou moins définitifs, d'acteurs à l'intersection entre la scène politique instituée et l'espace protestataire. Le 18 décembre 2011, après l'annonce des résultats des législatives de novembre 2011, c'est Al Adl, une organisation puissante et totalement à la marge de la politique instituée, qui annonce son retrait du M20.

\section{Des interactions qui affaiblissent le M20}

Alors même que le M20 a associé les conditions de son succès à sa capacité à dissoudre les identités particulières de ses composantes et à construire un «nous » vingt-févriériste face à un « eux » makhzénien, l'offre de réforme et les pressions exercées sur le M20 contribuent à décomposer ce «nous ». Outre les défections, les membres de la coalition se sentent assaillis autant par le Makhzen officiel que par ce qu'ils perçoivent comme un « Makhzen intérieur » à géométrie variable, composé de « cooptés » et d' « infiltrés ».

Vécus comme une reconnaissance du M20, les dispositifs mobilisés par la monarchie pour faire sortir le débat de la rue et l'acheminer vers les lieux de la politique instituée contribuent à étendre la protestation. Mais, dans la mesure où ils favorisent l'expression des divergences à un niveau plus horizontal (au sujet de la question de la commanderie des croyants et de l'islamité de l'État, du statut de la langue amazigh, du statut de la femme, etc.), ils mettent

\footnotetext{
${ }^{58}$ Pratiques réprouvées par les codes diffusés par la mouvance islamiste au-delà des militants.

${ }^{59}$ Voir à ce sujet Daniel Gaxie, «Économie des partis et rétributions du militantisme », Revue française de science politique, 27 (1), février 1977, p. 123-154.
} 
à l'épreuve la tentative du M20 de construire un face-à-face « gouvernants » / « gouvernés » ${ }^{60}$. À un premier niveau, la Commission consultative pour la révision de la constitution (CCRC) est boycottée par les coordinations du M20, par l'AMDH, ATTAC et deux partis très actifs au sein du M20 (le PSU et Annahj) ; en revanche, ses invitations sont acceptées par une trentaine de partis, cinq grandes centrales syndicales et une douzaine d'ONG. Bien davantage, au moment même où le M20 connaît son heure de gloire, des partis participent aux consultations du CCRC tout en relâchant la pression sur leurs jeunesses qui continuent à défiler pendant les marches du M20. Certains y voient le commencement de l'inversion du processus de départ. Plutôt que de révolutionner leurs partis de l'intérieur, les vingt-févriéristes de ces organisations seraient devenus les instruments de leurs partis qui chercheraient ainsi à renforcer leur position dans la scène politique instituée, en donnant à voir leur capacité de nuisance dans l'espace protestataire. Le mouvement Baraka du PJD pour commencer et des Usfpistes du M20 après le discours royal du 17 juin (annonciateur du référendum constitutionnel) seront parmi les premiers à faire défection du M20, tout en brandissant la menace de le rejoindre à nouveau si le processus de réforme n'est pas conforme à leurs attentes. Soulignons au passage que les leaders de ces partis pensent être les mieux placés pour peser dans le processus de révision constitutionnelle et, à terme, contribuer à recomposer la scène politique instituée en leur faveur. Quant aux syndicats, ils conservent une attitude d'entre-deux ; des vingt-févriéristes en concluent que, depuis leur engagement dans la politique de « dialogue social » initiée en 1996, ces centrales ne sont plus disposées à mobiliser les masses ouvrières en appelant à des grèves générales comme en 1981 ou en 1990, et encore moins à bloquer le système de production et de distribution.

Parallèlement, disqualifier devient l'une des principales tâches du « Mouvement de la jeunesse du 9 mars » et des « jeunes royalistes », des contre-mouvements inédits au Maroc ${ }^{61}$. Quant aux services de sécurité, ils continuent à recourir à des techniques rodées. "Profiler» les acteurs du M20 doit permettre de les connaître intimement, de « monter des dossiers » en vue d'infiltrer, d'acheter, d'intimider, de jouer sur les contradictions internes du mouvement pour briser le front et ternir l'image extérieure de celui-ci. La suspicion ne tarde pas à s'instiller au sein du mouvement, conduisant à se méfier de personnes "trop bien sapées », dotées d'appareil photo trop sophistiqués, ou encore prenant des photos à la manière des « flics ».

En outre, les forces centrifuges sont perçues comme des « manœuvres policières ». Dès le mois de mars, elles s'expriment à travers la création du «Collectif des indépendants du Mouvement du 20 février ». À l'origine de cette initiative, le président d'une association de quartier accuse les " organisations » (hay'at) d'avoir constitué un "noyau dur » afin de se concerter dans les coulisses et d'exercer leur emprise sur le M20. Cet " enfant du quartier» (weld ad-derb) raconte qu'à chacune de ses expériences partisane, électorale, associative, des personnes ont « exploité sa popularité » locale pour « grimper ${ }^{62}$. À nouveau, il a le sentiment d'être dépossédé.

Plus que les défections et la répression, ce sont les «nuisances » des membres de ce collectif qui perturbent le mouvement et qui exacerbent la méfiance à l'égard du " Makhzen intérieur ». Ceux qui sont désormais surnommés les "baltagis ${ }^{63}$ de l'intérieur» sont

\footnotetext{
${ }^{60}$ Voir J.-N. Ferrié, B. Dupret, «La nouvelle architecture constitutionnelle... », art. cité. Plus généralement, sur les «apprentissages » réalisés par les régimes de la région suite à la chute de Ben Ali puis de Moubarak, voir Steven Heydemann, Reinoud Leenders, « Authoritarian Learning and Authoritarian Resiliance », Globalizations, 8, 2011, p. 647-653.

${ }^{61}$ Pour la première fois, les « royalistes » sont visibilisés à travers les réseaux sociaux et les contre-manifestations, non comme une incarnation fusionnelle du «peuple », mais comme une composante en antagonisme avec un mouvement qui s'attaque aux prérogatives royales.

${ }^{62}$ Entretien, 21 novembre 2011.

${ }^{63}$ Depuis janvier 2011, ce mot désigne en Egypte les « fiers-à-bras et voyous » recrutés par les services de sécurité pour intimider les manifestants et les opposants.
} 
publiquement accusés d'être des agents des " services », du ministère de l'Intérieur ou des élus locaux. Les AG se prolongent de plus en plus tard dans un climat d'affrontement verbal, voire physique. De manière quasi rituelle, à chaque fois que les tensions montent au sein de l'AG, les participants lancent deux slogans en particulier. L'un s'accompagne d'un geste désignant l'adversaire intérieur: "Makhzen dégage » (wa al-makhzen ytle barra). Le second tente de rétablir le calme: "Unis et solidaires, nous obtiendrons ce que nous voulons". Rétrospectivement, des militants réalisent que ces "perturbations » ont canalisé toutes les énergies ${ }^{64}$.

\section{Les effets internes des batailles contre le Makhzen}

Selon notre hypothèse, les batailles menées par le M20-Casablanca contre le Makhzen officiel et le « Makhzen intérieur » de même que les réorientations imaginées pour compenser les défections contribuent peu à peu à modifier les équilibres initiaux au sein de la coalition, à consolider la position des plus dotés en capitaux militants et, à moyen terme, à attiser les feux de la discorde.

Tout d'abord, des militants « organisées » du M20, surnommés le « noyau dur », prennent vite l'habitude de se concerter en dehors des AG pour « fortifier » et « protéger » le mouvement. La volonté même de "réussir la marche du 20 mars » conduit à la mobilisation intensive des compétences militantes, des ressources humaines et logistiques des organisations les plus aguerries, ne serait-ce que pour mener les campagnes de diffusion de tracts, pour assurer le jour de la marche un service d'ordre comptant près de huit cents personnes et pour mettre en place un dispositif sonore et organisationnel d'unification du cortège (Photographie 1). Là où les uns et les autres s'émerveillent devant l'organisation de cette fameuse marche, une vidéaste éprouve le sentiment que les « jeunes du M20 sont dépossédés ». Dans l'ensemble, ce jour-là, se dégage l'impression que les militants d'Al Adl ont «montré leurs muscles ». Rétrospectivement, des membres du M20 relèvent qu'à l'exception d'ATTAC les composantes de gauche leur auraient délégué « par paresse » de plus en plus de tâches liées à la logistique à partir de cette date. Au cours des mois à venir et surtout pendant l'été 2011, le malaise qu'éprouvent surtout des « indépendants » du M20, face à une " organisation quasi paramilitaire » des sorties du M20, va en s'amplifiant.

Ensuite, pour compenser les premières défections et mener la « guerre du nombre » avant le référendum constitutionnel, l'AG du 3 mai décide de déplacer les marches du centre-ville vers les quartiers populaires. D'autres considérations justifient un tel changement aux yeux de la plupart des militants du "noyau dur» : sortir hebdomadairement au centre-ville devient « monotone » et ne crée plus de surprise; il importe de faire entendre la voix du mouvement dans tous les quartiers pour atteindre ceux qui n'ont jamais entendu parler de Facebook ; la mobilisation peut être plus «efficace» dans des quartiers denses. Certains d'entre eux réajustent leurs perceptions des similarités avec l'Égypte et considèrent désormais que le M20 se trouve dans la même position que le mouvement égyptien Kifaya en 2004 ; descendre dans les quartiers est une occasion rêvée pour « s'enraciner au sein du peuple » et élargir ses bases populaires afin de mieux préparer les luttes à venir. Un tel choix suscite des tensions internes, notamment après la répression du 22 et du 29 mai ${ }^{65}$. Des membres «non organisés », de la gauche gouvernementale, voire du PSU y voient une «provocation du Makhzen » par les militants d'Al Adl et des autres composantes de la gauche radicale qui menaceraient ainsi d'allumer le feu au sein des quartiers; ce qui aurait pour conséquence de démobiliser les hommes d'affaires et les « classes moyennes » impliqués dans le M20. Ils accusent précisément

\footnotetext{
${ }^{64}$ Entretien, 18 septembre 2011.

${ }^{65}$ Pendant ces épisodes, les forces de sécurité évitent de faire des morts. Pour la période allant du 20 février au 27 octobre 2011, l’AMDH dénombre dix « martyrs du Mouvement du 20 février ».
} 
Al Adl d'organiser des marches dans ses bastions et par là même d'accroître sa mainmise sur le M20. Plus globalement, des militants interprètent la répression du mois de mai comme une réaction des autorités, effrayées de voir le M20 manifester dans des lieux «difficiles à contrôler »; le spectre des émeutes de 1981 est brandi. Ils y perçoivent aussi la volonté de mettre fin à la mobilisation avant le référendum constitutionnel et les vacances d'été.

Sur un autre plan, les phases répressives du mois de mai affectent les performances du groupe. Elles le désorganisent, favorisent l'expression de la « spontanéité » et le surgissement de nouvelles hiérarchies. Des militants ne manquent pas de souligner : « Dès que les coups font reculer les caméras, ceux qui aiment se pavaner se mettent en retrait ». Comme pendant la répression du 13 mars $^{66}$, la « radicalisation » des slogans est d'abord impulsée par la dynamique propre de l'action protestataire. L'absence de dispositif de sonorisation sophistiqué accentue le caractère réactif des slogans scandés par des militants hissés sur les épaules des plus costauds. La radicalisation est également liée à la nouvelle configuration de la coalition du M20 ; un membre du comité des slogans de la gauche non gouvernementale explique qu'il a cessé de s'autolimiter dès que l'USFP a annoncé sa participation au référendum constitutionnel : «c'est pour les épargner qu'on évitait certains slogans $»{ }^{67}$. Pendant cette phase, la tonalité transgressive à l'égard du roi s'amplifie de manière inédite. Mais dès le mois de juin, face aux réactions internationales suscitées par la répression du mois de mai, les autorités privilégient la « sous-traitance de la répression ${ }^{68}$.

Défections, lutte contre le Makhzen « extérieur » et « intérieur », répression alimentent deux dynamiques. D'une part, les options privilégiées par le M20 sont interprétées par un ensemble d'acteurs au sein et en dehors du mouvement comme des signes de « radicalisation ", et ces perceptions engendrent à leur tour de nouvelles sources de tensions internes. D'autre part, les positions des plus dotés en capitaux militants de même que leur complicité se consolident au sein de la coalition au détriment des " indépendants »; Adlistes et militants d'une partie de la gauche radicale se perçoivent mutuellement comme des « personnes sûres », appartenant à des organisations « qui ont payé, qui paient et qui paieront le prix » de leur engagement au sein du M20 ${ }^{69}$. Mais c'est surtout dans un processus de « dé-assurance » et de contre « bandwagon effect » que les conflits internes atteignent leur paroxysme.

\section{"Dé-assurance ", contre "bandwagon effect " et démoralisation}

Pendant les séquences allant de février à avril 2011, nous avons observé les effets du « jeu d'assurance » et du «bandwagon effect» : le processus lancé par les pionniers du M20 et, préalablement, par les pionniers du «Printemps arabe » se renforce grâce au ralliement de suiveurs qui participent au mouvement après avoir observé et/ou anticipé ses succès. Inversement, les séquences suivantes se traduisent par le phénomène opposé : le sentiment que les chances de succès du mouvement se sont amenuisées contribue peu à peu à détacher des wagons du train en marche.

En premier lieu, l'adoption de la nouvelle constitution, après le référendum du $1^{\text {er }}$ juillet 2011, constitue un coup très dur pour les vingt-févriéristes. À partir de cette date, certains reconnaissent que « le mouvement a perdu sa capacité à orienter le débat du fait de la machine de guerre mise en place par le Makhzen, dont nous avons sous-estimé la sophistication ${ }^{70}$.

\footnotetext{
${ }^{66}$ Ce jour-là, dès que les coups ont commencé à pleuvoir, le slogan qui réclame « un roi qui règne mais qui ne gouverne pas » devient : « un roi qui ne règne pas et qui ne gouverne pas ».

${ }^{67}$ Entretien, septembre 2011.

${ }^{68}$ Par là, les militants du M20 font référence aux attaques (jets de pierre, arme blanche) perpétrées par des « voyous » qu'ils accusent d'être recrutés par les autorités ou des élus locaux.

${ }^{69}$ Entretien avec un Adliste, juillet 2011.

${ }^{70}$ Entretien avec un blogueur, juillet 2011.
} 
Lorsque les médias nationaux ne boudent pas le M20, ils annoncent sa mort imminente, alors même que le mouvement organise à Casablanca des marches estivales plus mobilisatrices que jamais (près de 80000 manifestants selon les organisateurs).

Sur un autre plan, les vingt-févriéristes ont l'impression que ce qui se passe à l'échelle régionale et internationale les dessert. Les discours internationaux prédominants érigent les voies de la réforme empruntées par la monarchie en alternative aux options révolutionnaires. L’Union européenne et le G8 soulignent la nécessité de soutenir les " progrès encourageants » réalisés dans des pays comme le Maroc. Des « amis du roi » sont invités à représenter le Maroc dans des universités d'été européennes consacrées aux révolutions arabes. Par ailleurs, des interviewés ont le sentiment que les images de guerre civile et de répression sanglante en Libye et en Syrie, de même que les difficultés de la reconstruction en Tunisie et en Égypte, exercent des effets dissuasifs au sein de la population marocaine.

Peu à peu, la démoralisation gagne du terrain parmi les vingt-févriéristes, et ce en dépit des tentatives de redynamiser le mouvement et des batailles qui lui donnent un nouveau souffle (les marches imposantes des soirées estivales du ramadan, la campagne de mobilisation pour libérer le rappeur du M20, la campagne de boycott des législatives de novembre 2011). L'épuisement s'installe d'autant plus que les succès tangibles tardent à venir, que le sentiment de vivre un moment historique s'émousse, que certains ont l'impression que les pratiques « fossiles » des organisations ont brisé l'élan innovateur du M20 et, surtout, que les combats menés par le mouvement bénéficient à des composantes en particulier.

En effet, après le référendum du $1^{\text {er }}$ juillet, les conflits internes s'exacerbent au sein de la coordination du M20 à Casablanca. Ils se publicisent essentiellement pendant les AG et dans les échanges sur Facebook; les médias ne manquent pas de les répercuter à leur manière. Ils s'expriment dans des registres aussi variés que l'argumentation, la disqualification, l'insulte et la violence physique, pourtant condamnée par le mouvement, avec une constante de l'histoire protestataire marocaine : quelle que soit son identité politique, l'ennemi intérieur est systématiquement accusé d'être un agent du Makhzen. Si le ton n'est plus à l'atténuation des différences « culturelles » entre « gauchistes $\rangle^{71}$ et $~ «$ islamistes », la principale ligne de partage est autre. Les militants du PSU et des «indépendants » réclament un « débat d'idées » et attendent d'Adl et des autres composantes de la gauche radicale qu'ils « rassurent les classes moyennes » en exprimant clairement leur adhésion à la monarchie parlementaire. En revanche, les principaux membres du " noyau dur » (des militants d'Al Adl et des autres composantes de la gauche radicale) refusent de fixer un «seuil » au mouvement et considèrent que le débat idéologique provoque des dissensions, qu'il faut se concentrer sur ce qui unit le mouvement, c'est-à-dire l'action sur le terrain. Sur un autre plan, en été, tous ceux qui prônent une culture politique alternative reprochent au «noyau dur » de continuer à pratiquer « les coulisses » et à contourner l'AG, principal rouage des pratiques de démocratie participative, alors même que les «baltagis de l'intérieur» sont neutralisés. Plus que jamais, ils accusent les Adlistes de recevoir des ordres de leur hiérarchie et d'imposer leur hégémonie sur le plan organisationnel, avec la complicité d'une partie des militants de la gauche radicale. Ils perçoivent les signes de cette prise en main à travers des détails et des incidents : des absences remarquées pendant les ateliers de discussion impulsés par des " indépendants », le choix du calendrier des marches pendant le ramadan, le fait que des Adlistes empêchent des jeunes filles dont ils n'apprécient pas le «look» de monter dans le véhicule sonorisé, qui permet d'organiser le cortège, etc. Certains vont jusqu'à déceler « la prise de pouvoir » dans la manière de déambuler pendant les marches de leur responsable : « on dirait le maître de céans pendant une cérémonie de mariage ( $m u l$ al-'ars)». Bien plus, les variations que connaît le nombre des manifestants sont interprétées tantôt comme " une descente » d'Al Adl tantôt comme « un retrait », avec le même

\footnotetext{
${ }^{71}$ Qualification revendiquée par quelques « indépendants ».
} 
but à chaque fois : démontrer la centralité de l'organisation à ses adversaires au sein du M20. L'ensemble de ces accusations sont rejetées par les membres du «noyau dur », qui se sont réappropriés cette exolabellisation. D'après un Adliste, «les accusations d'hégémonie [de certains vingt-févriéristes de gauche] ne sont que le reflet de leur peur : ils voient les islamistes l'emporter en Libye, en Tunisie, puis en Égypte...» ${ }^{72}$.

Peu après la victoire du PJD aux législatives du 25 novembre 2011, Al Adl annonce son retrait du M20 à l'échelle nationale. Certains y voient une main tendue aux « frères islamistes ". D'autres y perçoivent le refus d'Al Adl de continuer de s'investir dans une coalition où des composantes veulent fixer un seuil au mouvement. Mais par-delà le communiqué officiel, les entretiens réalisés montrent plutôt que les responsables d'Al Adl ont le sentiment que leurs " sacrifices » ont bénéficié au PJD et que le peuple n'est pas encore « mûr ». La défection de cette puissante organisation produit des effets tant sur les performances de ce qui reste du M20 à Casablanca que sur la recomposition de la coalition.

Les marches hebdomadaires continuent à être organisées et à attirer des porteurs de demandes sociales plus ou moins particularistes ; elles ramènent ponctuellement dans le giron du M20 des militants qui l'ont quitté pour sanctionner «sa radicalisation sous l'égide d'Al Adl ». Toutefois, le nombre des participants se réduit comme une peau de chagrin. Par ailleurs, si le dispositif organisationnel mis en place initialement permettait d'unifier la marche, de contenir les forces centrifuges, de privilégier des slogans à faible teneur idéologique, à partir du 25 décembre 2011, les identités idéologiques surgissent au grand jour, se disputant l'espace sonore et visuel. C'est particulièrement visible lors de la marche du $1^{\text {er }}$ janvier 2012. Au sein du cortège officiel du M20, slogans et pancartes prennent une tonalité de gauche et les photos des martyrs de la gauche des années de plomb pullulent (Photographie 2). Dans les marges, les familles des détenus salafistes, encadrées par des membres du Collectif des indépendants, se permettent de manière inédite de scander des slogans à caractère religieux et de brandir une banderole sur laquelle « Dieu est grand » est calligraphié. Enfin, le $1^{\text {er }}$ janvier 2012, le slogan « Le peuple veut la chute du régime » commence à être scandé du haut du véhicule sonore officiel de la coordination du M20-Casablanca.

Photographie 2 C M. Bennani-Chraïbi

Marche du $1^{\text {er }}$ janvier 2012 à Derb Ghallef à Casablanca. En avant plan, la calligraphie « Dieu est grand» sur une banderole. En arrière plan, des photographies des « martyrs » de gauche.

\footnotetext{
${ }^{72}$ Entretien, novembre 2011.
} 


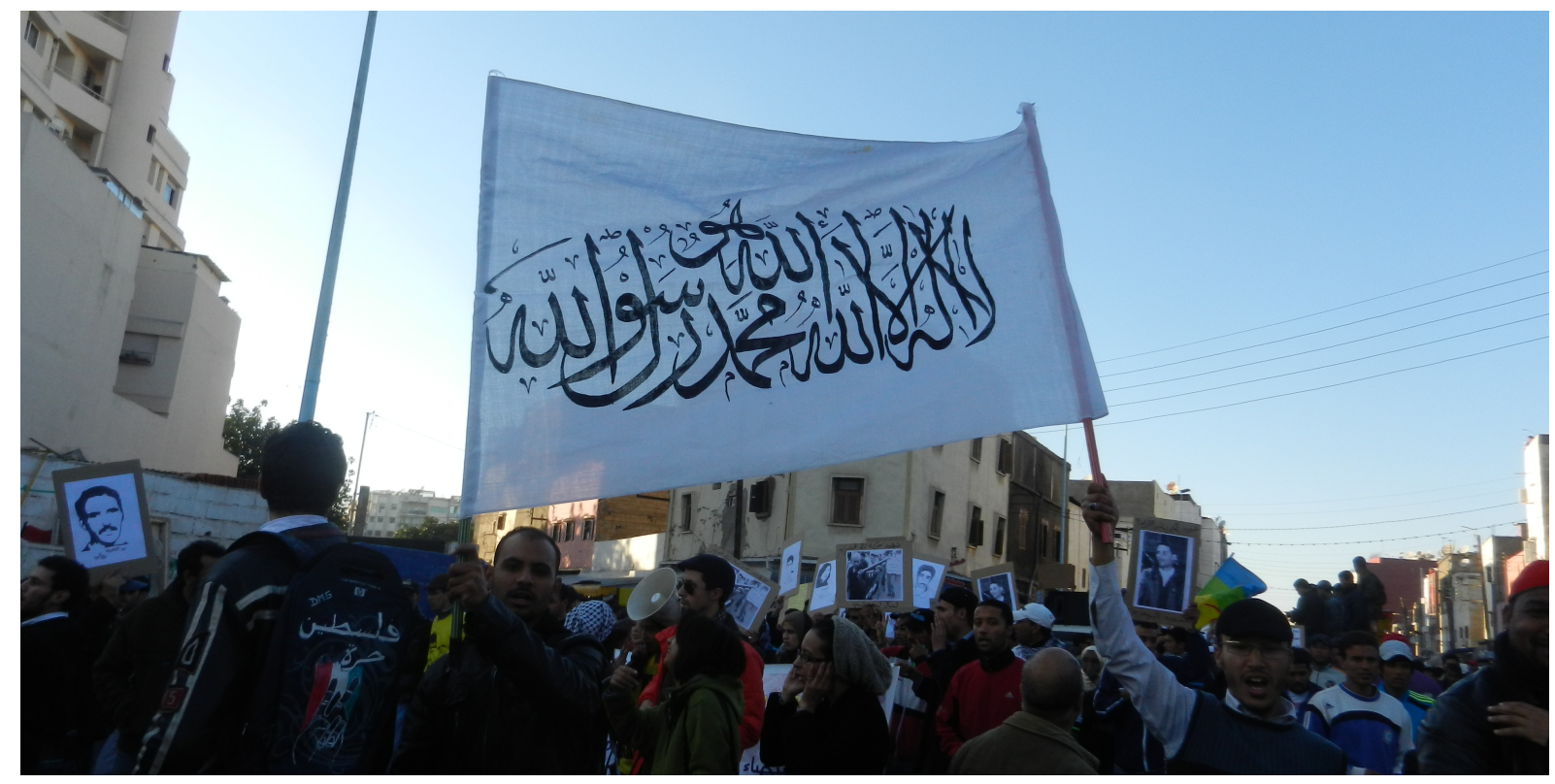

Au niveau de la coalition du M20-Casablanca, la défection d'Al Adl ne conduit ni à inverser le rapport de force entre «indépendants » et «organisés », ni à «modérer» le mouvement. D'une part, elle constitue une inflexion décisive dans le processus de reconfiguration du M20 qui observe le rétrécissement du collectif autour des noyaux de la gauche radicale. D'autre part, elle anéantit les efforts d'autolimitation visant à préserver l'unité de la coalition et à maintenir un seuil de revendications en phase avec l'appréciation de la conjoncture et des rapports de force. En effet, grâce à leur discipline, à leurs capacités organisationnelles et mobilisatrices, les militants d'Al Adl parvenaient à tenir aussi bien la rue que la ligne du «noyau dur ». Autrement dit, ils contribuaient à canaliser le mouvement et d'une certaine manière à le « modérer ».

$$
* * *
$$

Le 21 février 2012, dans un post sur FB, H.A qui a contribué à rédiger le premier appel du M20 invite à interrompre l'expérience : «Les dinosaures ont transformé le M20 en quelque chose qui ne diffère que par le nom des mouvements qui ont échoué dans le passé. Sous de nouvelles formes, ils ont commis les mêmes erreurs, produit les mêmes illusions. Ceux qui disent que le mouvement perdure ne parlent que de la continuité du label...»

Après un an d'échanges de coups avec les autorités et au sein de l'espace protestataire, le M20 ressort affaibli. Loin d'être le produit d'un effet domino, il est tributaire d'un processus d'identification et d'attribution de similarité, de la réactivation de relais organisationnels et de réseaux plus ou moins dormants. Une large coalition regroupe néophytes et militants aguerris, au sein d'un champ d'alliance et d'opposition à la jonction entre scène politique instituée et espace protestataire, par-delà les clivages entre réseaux de gauche et islamistes. Dans un jeu d'échelles entre l'interne, le local, le national, le régional et l'international, un faisceau d'actions, d'interactions et d'événements contribue autant à l'enracinement de la coalition qu'à sa désagrégation. La protestation se déploie et se régénère en puisant dans plusieurs registres : la réactivité du régime ; le souci de celui-ci de préserver sa position de premier de la classe de la région ; la croyance des protestataires en leur capacité à gagner le Makhzen ; leur sentiment que l'occasion est historique et que le succès est à portée de main ; les gratifications et les effets surgénérateurs de l'engagement; les dispositifs mis en place pour maintenir la coalition et masquer les identités particularistes. 
Quant à la désagrégation progressive de la coalition, elle chemine de manière visible ou souterraine, en lien avec des interactions intentionnelles et non intentionnelles. Les premières défections sont le fait d'acteurs suffisamment bien positionnés sur la scène politique instituée pour espérer peser sur sa recomposition et bénéficier des réformes amorcées. Sur un autre plan, l'infiltration et l'exacerbation de la suspicion à l'égard d'un « Makhzen intérieur » brouillent les frontières entre «nous» et " eux», enrayant ainsi le processus de polarisation. Bien davantage, la répression, les batailles menées contre le Makhzen " intérieur » et " extérieur », les tentatives de compenser les défections favorisent, au sein de la coalition reconfigurée, l'hégémonie des acteurs les plus dotés en capitaux militants au détriment de ceux qui aspirent à faire de la politique autrement. Le coup de grâce est donné par la deuxième grande série de défections, marquée par le retrait de l'organisation islamiste, considérée comme la plus puissante dans l'espace protestataire. Il se produit dans une atmosphère de démoralisation, ponctuée par les perceptions suivantes : l'impression d'avoir "perdu la bataille » dans une conjoncture régionale dissuasive ; la perception d'une décélération de l'histoire ; l'érosion du sentiment de constituer un "nous » soudé dans l'adversité; la croyance que les fruits de l'engagement sont récoltés par des composantes au détriment d'autres. À chaque fois que les défections privent la coalition du M20 des segments qui contribuent à la «modérer» ou à la « tenir », le processus de radicalisation s'amplifie d'un cran au sein d'un collectif où ne restent plus que ceux qui ne perçoivent pas d'autre alternative à l'occupation de la rue. Dans le cas observé, "l'incertitude structurelle » intrinsèque aux conjonctures fluides est rapidement compensée par l'autolimitation qui prévaut aussi bien chez les autorités publiques que dans un mouvement protestataire dominé par des acteurs " organisés », rétifs aux débordements et aux dérapages, fiers de leur capacité à tenir la rue, attentifs à la portée de chacun de leurs coups. Aussi, la radicalisation se nourrit-elle davantage de l'affaiblissement du mouvement que de sa force ou du dépassement des pionniers par des suiveurs imprévisibles.

En février 2012, les foyers de protestation se développent au-delà du M20 tout en prenant d'autres formes : une poursuite des mobilisations sectorielles; une banalisation du slogan « Dégage »; des explosions de violence montrant à quel point l'autolimitation est une option précaire ; un «empiètement silencieux du quotidien " ${ }^{73}$ à travers la conquête des espaces urbains par les vendeurs ambulants et l'expansion de la construction d'habitats irréguliers. Au moment même où la coordination du M20 commence à s'affaiblir, ses chevilles ouvrières prennent conscience qu'elles ont ouvert une boîte de pandore et un responsable des forces de sécurité va jusqu'à nous confier ${ }^{74}$ : «plus jamais ce ne sera pareil, les citoyens n'ont plus le même rapport à l'autorité $»^{75}$.

\footnotetext{
${ }^{73}$ Asef Bayat, Street Politics. Poor People Movements in Iran, Cambridge, Cambridge University Press, 1997.

${ }^{74}$ Entretien, février 2012.

75 Toute notre gratitude va aux membres du M20 à Casablanca pour l'accueil réservé à notre enquête. Nous remercions également Philippe Blanchard, Dina El Khawaga, Olivier Fillieule, Choukri Hmed et les évaluateurs de la revue pour leur lecture stimulante.
} 